\title{
ON WELL-POSEDNESS, REGULARITY AND EXACT CONTROLLABILITY FOR PROBLEMS OF TRANSMISSION OF PLATE EQUATION WITH VARIABLE COEFFICIENTS
}

\author{
BY \\ BAO-ZHU GUO (Academy of Mathematics and Systems Science, Academia Sinica, Beijing 100080, \\ P.R. China and School of Computational and Applied Mathematics, University of the Witwatersrand, \\ Wits 2050, Johannesburg, South Africa) \\ AND
}

ZHI-CHAO SHAO (School of Computational and Applied Mathematics, University of the Witwatersrand, Wits 2050, Johannesburg, South Africa)

\begin{abstract}
A system of transmission of Euler-Bernoulli plate equation with variable coefficients under Neumann control and collocated observation is studied. Using the multiplier method on a Riemannian manifold, it is shown that the system is well-posed in the sense of D. Salamon. This establishes the equivalence between the exact controllability of an open-loop system and the exponential stability of a closed-loop system under the proportional output feedback. The regularity of the system in the sense of G. Weiss is also proved, and the feedthrough operator is found to be zero. These properties make this PDE system parallel in many ways to the finite-dimensional ones. Finally, the exact controllability of an open-loop system is developed under a uniqueness assumption by establishing the observability inequality for the dual system.
\end{abstract}

1. Introduction and main results. In distributed parameter control systems, many properties are closely related to each other. In the early 1970's, Russell found that for some time-invertible PDEs, the exponential stability of a closed-loop system can deduce the exact controllability of an open-loop system $([24,25])$. Conversely, it was also found that the exact controllability of an open-loop system implies the exponential stability of a closed-loop system provided that the open-loop system is well-posed in the sense of D. Salamon ([2, 11]). Lagnese [17] studied some LQ-optimal control problems

Received June 15, 2006.

2000 Mathematics Subject Classification. Primary 35L35, 93C20, 93D15, 93B05, 93B07.

Key words and phrases. Euler-Bernoulli plate equation, well-posedness and regularity, boundary control and observation, exact controllability, exact observability, multiplier method on Riemannian manifold. This work was carried out with the support of the National Natural Science Foundation of China and the National Research Foundation of South Africa. Zhi-Chao Shao acknowledges the support of the Postdoctoral Fellowship of the Claude Leon Foundation of South Africa.

E-mail address: bzguo@iss.ac.cn

E-mail address: zcshao@cam.wits.ac.za 
for beam equations to obtain the stability of the system. For the general result from optimizability to exponential stability, we refer to [30].

In the past two decades, it has been discovered that the well-posed and regular systems are a quite general class of linear infinite-dimensional systems, and that they cover many control systems described by partial differential equations with actuators and sensors supported on isolated points, sub-domains, or on parts of the boundary of the spatial region ([8]). This class of infinite-dimensional systems, although the input and output operators are allowed to be unbounded, may possess many properties that are parallel in many ways to finite-dimensional systems ([9]).

Although the abstract theory of well-posed and regular linear systems has been quite fruitful, only a few multi-dimensional PDEs have been verified to be well-posed and regular. For the systems with constant coefficients, the well-posedness and regularity of a multi-dimensional heat equation with both Dirichlet and Neumann type boundary controls were established in [4] and that for a wave equation with boundary Dirichlet input and collocated output was proved in [1] and [12]. The well-posedness and regularity for the multi-dimensional Schrödinger equation and the Euler-Bernoulli equation were reported in $[19,13,14]$. Very recently, these results were generalized to multidimensional wave and plate equations with variable coefficients under boundary controls and observations $([15,16])$.

In this paper, we study the following system of transmission of the Euler-Bernoulli plate equation with variable coefficients under Neumann control and collocated observation:

$$
\left\{\begin{array}{l}
\ddot{w}(x, t)+a(x) \mathcal{A}^{2} w(x, t)=0, \quad(x, t) \in Q, \\
w(x, 0)=w^{0}(x), \dot{w}(x, 0)=w^{1}(x), \quad x \in \Omega, \\
w_{2}(x, t)=0, \frac{\partial w_{2}}{\partial \nu_{\mathcal{A}}}(x, t)=u(x, t), \quad(x, t) \in \Sigma, \\
w_{1}(x, t)=w_{2}(x, t), \frac{\partial w_{1}}{\partial \nu_{\mathcal{A}}}(x, t)=\frac{\partial w_{2}}{\partial \nu_{\mathcal{A}}}(x, t), \quad(x, t) \in \Sigma_{1}, \\
a_{1} \mathcal{A} w_{1}(x, t)=a_{2} \mathcal{A} w_{2}(x, t), a_{1} \frac{\partial \mathcal{A} w_{1}}{\partial \nu_{\mathcal{A}}}(x, t)=a_{2} \frac{\partial \mathcal{A} w_{2}}{\partial \nu_{\mathcal{A}}}(x, t), \quad(x, t) \in \Sigma_{1}, \\
y(x, t)=-\mathcal{A}\left(\mathscr{A}^{-1} \dot{w}(x, t)\right),(x, t) \in \Sigma,
\end{array}\right.
$$

where $\Omega \subset \mathbb{R}^{n}(n \geq 2)$ is an open bounded region with boundary $\partial \Omega=\Gamma$ of $C^{4}$-class, $\Omega_{1}$ is a bounded region contained inside of $\Omega$ : $\bar{\Omega}_{1} \subset \Omega$ and $\Gamma_{1}=\partial \Omega_{1}$ is of $C^{4}$-class. $\Omega_{2}=\Omega \backslash \Omega_{1}, Q=\Omega \times(0, T), Q_{1}=\Omega_{1} \times(0, T), Q_{2}=\Omega_{2} \times(0, T), \Sigma=\Gamma \times(0, T)$, and $\Sigma_{1}=\Gamma_{1} \times(0, T), T>0$ is a time moment. $u$ is the input function (or control) and $y$ is the output function (or observation).

$$
\mathcal{A} w(x, t):=\sum_{i, j=1}^{n} \frac{\partial}{\partial x_{i}}\left(a_{i j}(x) \frac{\partial w(x, t)}{\partial x_{j}}\right), D(\mathcal{A})=H^{2}(\Omega) \cap H_{0}^{1}(\Omega)
$$


with $a_{i j}=a_{j i} \in C^{\infty}\left(\mathbb{R}^{n}\right)$ and for some constant $\alpha>0$,

$$
\begin{gathered}
\sum_{i, j=1}^{n} a_{i j}(x) \xi_{i} \overline{\xi_{j}} \geq \alpha \sum_{i=1}^{n}\left|\xi_{i}\right|^{2}, \forall x \in \Omega, \xi=\left(\xi_{1}, \xi_{2}, \cdots, \xi_{n}\right) \in \mathbb{C}^{n} \\
\mathscr{A} w=\mathcal{A}^{2} w, D(\mathscr{A})=H^{4}(\Omega) \cap H_{0}^{2}(\Omega) \\
\nu_{\mathcal{A}}:=\left(\sum_{k=1}^{n} \nu_{k} a_{k 1}, \sum_{k=1}^{n} \nu_{k} a_{k 2}, \cdots, \sum_{k=1}^{n} \nu_{k} a_{k n}\right), \frac{\partial}{\partial \nu_{\mathcal{A}}}:=\sum_{i, j=1}^{n} \nu_{i} a_{i j} \frac{\partial}{\partial x_{j}}
\end{gathered}
$$

with $\nu=\left(\nu_{1}, \nu_{2}, \cdots, \nu_{n}\right)$ the unit normal vector on $\Gamma$ or $\Gamma_{1}$ pointing toward the exterior of $\Omega_{2} . w_{1}=\left.w\right|_{\Omega_{1}}, w_{2}=\left.w\right|_{\Omega_{2}}, \dot{w}=w_{t}$, and $a(x)$ is given by

$$
a(x)=\left\{\begin{array}{l}
a_{1}, x \in \Omega_{1}, \\
a_{2}, x \in \Omega_{2},
\end{array}\right.
$$

where $a_{1}, a_{2}$ are positive constants.

When $a_{1}=a_{2}$ the system (1.1) is reduced to the ordinary plate equation with variable coefficients studied in $[16,34]$. When $a_{1} \neq a_{2}$, the exact controllability for the system (1.1) with constant spatial coefficients and additional lower-order terms on the right hand side of the governing equation (1.1) was established in [21] (see also [3]). [23] studied the uniform stability for the solutions of a transmission problem in non-homogeneous anisotropic elasticity. The stabilization of a thermoelastic plate with variable coefficients can be found in [6]. For the exact controllability and uniform stabilization of problems of transmission for wave and shell equations, we refer to [5, 7, 18, 22].

One of the aims of this paper is to establish the equivalence between the exact controllability of open-loop system (1.1) and the exponential stability of its closed-loop form under the proportional feedback $u=-k y, k>0$. This is a consequence of the wellposedness for the system (1.1). The well-posedness together with the regularity property will put the system (1.1) into a general framework of well-posed and regular linear infinite-dimensional systems that has been studied extensively in the past two decades $([8])$.

$$
\text { Let } \mathcal{H}=L^{2}(\Omega) \times H^{-2}(\Omega) \text { and } U=L^{2}(\Gamma) \text {. }
$$

Theorem 1.1. Let $T>0,\left(w^{0}, w^{1}\right) \in \mathcal{H}$ and $u \in L^{2}(0, T ; U)$. Then there exists a unique solution $(w, \dot{w}) \in C([0, T] ; \mathcal{H})$ to Equation (1.1). Moreover, if $a_{2} \leq a_{1}$, then there exists a constant $C_{T}>0$, independent of $\left(w^{0}, w^{1}, u\right)$, such that

$$
\|(w(\cdot, T), \dot{w}(\cdot, T))\|_{\mathcal{H}}^{2}+\|y\|_{L^{2}(0, T ; U)}^{2} \leq C_{T}\left[\left\|\left(w^{0}, w^{1}\right)\right\|_{\mathcal{H}}^{2}+\|u\|_{L^{2}(0, T ; U)}^{2}\right] .
$$

Theorem 1.1 implies that the open-loop system (1.1) is well-posed provided that $a_{2} \leq$ $a_{1}$ in the sense of D. Salamon with the state space $\mathcal{H}$, input and output space $U([27])$. From this result and $[2,11]$, we immediately have the following Corollary 1.1.

Corollary 1.1. Assume that $a_{2} \leq a_{1}$. Then the system (1.1) is exactly controllable in $\mathcal{H}$ on $[0, T]$ for some $T>0$ if and only if its closed-loop system under the proportional output feedback $u=-k y(k>0)$ is exponentially stable. 
Theorem 1.2. Assume that $a_{2} \leq a_{1}$. Then the system (1.1) is regular in the sense of G. Weiss with zero feedthrough operator. Precisely, if $w(\cdot, 0)=\dot{w}(\cdot, 0)=0$ and $u(\cdot, t) \equiv u(\cdot) \in U$ is a step input, then the corresponding output $y$ satisfies

$$
\lim _{\sigma \rightarrow 0} \int_{\Gamma_{0}}\left|\frac{1}{\sigma} \int_{0}^{\sigma} y(x, t) d t\right|^{2} d x=0 .
$$

Theorems 1.1 and 1.2 ensure that the system (1.1) is a well-posed regular linear system. This makes the system (1.1) parallel in many ways to a linear finite-dimensional system.

It should be pointed out that due to variable coefficients, the classical multiplier method in Euclidean space seems inadequate to prove Theorems 1.1 and 1.2. Some computations on the Riemannian manifold are needed as in [34].

Because of the well-posedness claimed by Theorem 1.1, the exact controllability of system (1.1) can be obtained by establishing the observability inequality for the dual system (5.1). To do this, we need some additional assumptions (H1)-(H3) stated below.

Notice the condition (1.3) and let $A(x)$ and $G(x)$ be, respectively, the coefficient matrix and its inverse:

$$
A(x):=\left(a_{i j}(x)\right), G(x):=\left(g_{i j}(x)\right)=A(x)^{-1}, \mathcal{G}(x):=\operatorname{det}\left(g_{i j}(x)\right) .
$$

For each $x=\left(x_{1}, x_{2}, \cdots, x_{n}\right) \in \mathbb{R}^{n}$, define the inner product and norm over the tangent space $\mathbb{R}_{x}^{n}$ of $\mathbb{R}^{n}$ by

$$
\begin{aligned}
& g(X, Y):=\langle X, Y\rangle_{g}=\sum_{i, j=1}^{n} g_{i j} \alpha_{i} \beta_{j}, \\
& |X|_{g}:=\langle X, X\rangle_{g}^{1 / 2}, \forall X=\sum_{i=1}^{n} \alpha_{i} \frac{\partial}{\partial x_{i}}, Y=\sum_{i=1}^{n} \beta_{i} \frac{\partial}{\partial x_{i}} \in \mathbb{R}_{x}^{n} .
\end{aligned}
$$

Then $\left(\mathbb{R}^{n}, g\right)$ is a Riemannian manifold with Riemannian metric $g([34])$. Denote by $D$ the Levi-Civita connection with respect to $g$. Let $N$ be a vector field on $\left(\mathbb{R}^{n}, g\right)$. Then for each $x \in \mathbb{R}^{n}$, the covariant differential $D N$ of $N$ determines a bilinear form on $\mathbb{R}_{x}^{n}$ :

$$
D N(X, Y)=\left\langle D_{X} N, Y\right\rangle_{g}, \forall X, Y \in \mathbb{R}_{x}^{n},
$$

where $D_{X} N$ stands for the covariant derivative of the vector field $N$ with respect to $X$.

Assumption (H1). There exists a vector field $N$ on $\left(\mathbb{R}^{n}, g\right)$ such that

$$
D N(X, X)=b(x)|X|_{g}^{2}, \forall X \in \mathbb{R}_{x}^{n}, x \in \Omega,
$$

where $b(x)$ is a function defined on $\Omega$ so that

$$
b_{0}=\inf _{x \in \Omega} b(x)>0 .
$$

Assumption (H2). There exists a vector field $N$ on $\left(\mathbb{R}^{n}, g\right)$ such that

$$
N(x) \cdot \nu \leq 0, x \in \Gamma_{1} .
$$


Assumption (H3) [the uniqueness assumption]. The following problem

$$
\left\{\begin{array}{l}
a(x) \mathcal{A}^{2} u=\zeta u \text { in } \Omega, \\
u_{2}=\frac{\partial u_{2}}{\partial \nu_{\mathcal{A}}}=0 \text { on } \Gamma \\
u_{1}=u_{2}, \frac{\partial u_{1}}{\partial \nu_{\mathcal{A}}}=\frac{\partial u_{2}}{\partial \nu_{\mathcal{A}}} \text { on } \Gamma_{1}, \\
a_{1} \mathcal{A} u_{1}=a_{2} \mathcal{A} u_{2}, a_{1} \frac{\partial \mathcal{A} u_{1}}{\partial \nu_{\mathcal{A}}}=a_{2} \frac{\partial \mathcal{A} u_{2}}{\partial \nu_{\mathcal{A}}} \text { on } \Gamma_{1}, \\
\mathcal{A} u_{2}=0 \text { on } \Gamma_{0},
\end{array}\right.
$$

possesses a unique zero solution, where $\zeta$ is an arbitrary complex number and $\Gamma_{0}$ is relatively open in $\Gamma$ defined by

$$
\Gamma_{0}=\{x \in \Gamma \mid N(x) \cdot \nu>0\} .
$$

When $a_{i j}(x)=\delta_{i j}$, then for some fixed $x_{0}$, the radial field $N=x-x_{0}$ meets Assumption (H1) with $b(x) \equiv 1$ and Assumption (H3) is a valid fact (Theorem 4.2 of [21]). For the variable coefficients case, several corollaries were presented in [35] to show how to verify Assumption (H1) by means of the Riemannian geometry method, but for Assumption (H3), it is not verified even for Euler-Bernoulli plate equations without transmission. As was indicated in [34], the problem is not a Cauchy problem, and hence many uniqueness theorems cannot be applied. We propose it as an unsolved problem here.

Theorem 1.3. Under Assumptions (H1)-(H3) and $a_{2} \leq a_{1}$, the system (1.1) is exactly controllable on some $[0, T], T>0$. That is, for any initial data $\left(w^{0}, w^{1}\right) \in \mathcal{H}$, there exists a control $u \in L^{2}(\Sigma)$ with $u=0$ on $\Sigma \backslash \Sigma_{0}$ such that the solution of (1.1) satisfies $w(\cdot, T)=w_{t}(\cdot, T)=0$.

The remainder of the paper is organized as follows. In Section 2, the system (1.1) is cast into an abstract setting studied in $[2,11]$ and some basic background on Riemannian geometry is introduced. The proof of Theorem 1.1 is given in Section 3. Section 4 is devoted to the proof of Theorem 1.2. Finally, in Section 5, we show the exact controllability under Assumptions (H1)-(H3).

2. Abstract formulation and preliminaries. Let $H=H^{-2}(\Omega)$. Let $A$ be the positive self-adjoint operator in $H$ induced by the bilinear form $\beta(\cdot, \cdot)$ defined by

$$
\langle A f, g\rangle_{H^{-2}(\Omega) \times H_{0}^{2}(\Omega)}=\beta(f, g)=\int_{\Omega} a(x) \mathcal{A} f(x) \cdot \overline{\mathcal{A} g(x)} d x, \forall f, g \in H_{0}^{2}(\Omega) .
$$

By means of the Lax-Milgram theorem, $A$ is a canonical isomorphism from $D(A)=$ $H_{0}^{2}(\Omega)$ onto $H$. Set

$$
\begin{aligned}
& H^{4}\left(\Omega, \Gamma_{1}\right)=\left\{w \in H_{0}^{2}(\Omega), w_{i} \in H^{4}\left(\Omega_{i}\right), i=1,2 \mid\right. \\
& \left.a_{1} \mathcal{A} w_{1}=a_{2} \mathcal{A} w_{2} \text { and } a_{1} \frac{\partial \mathcal{A} w_{1}}{\partial \nu_{\mathcal{A}}}=a_{2} \frac{\partial \mathcal{A} w_{2}}{\partial \nu_{\mathcal{A}}} \text { on } \Gamma_{1}\right\}
\end{aligned}
$$


with the norm

$$
\|w\|_{H^{4}\left(\Omega, \Gamma_{1}\right)}=\left(\left\|w_{1}\right\|_{H^{4}\left(\Omega_{1}\right)}^{2}+\left\|w_{2}\right\|_{H^{4}\left(\Omega_{2}\right)}^{2}\right)^{\frac{1}{2}} .
$$

Similar to Lemmas 1.1, 1.2 and 1.3 of [21], it can be shown that

$$
\left(\left\|w_{1}\right\|_{H^{4}\left(\Omega_{1}\right)}^{2}+\left\|w_{2}\right\|_{H^{4}\left(\Omega_{2}\right)}^{2}\right)^{\frac{1}{2}} \sim\left(\left\|\mathcal{A}^{2} w_{1}\right\|_{L^{2}\left(\Omega_{1}\right)}^{2}+\left\|\mathcal{A}^{2} w_{2}\right\|_{L^{2}\left(\Omega_{2}\right)}^{2}\right)^{\frac{1}{2}},
$$

and $H^{4}\left(\Omega, \Gamma_{1}\right)$ is dense in $H_{0}^{2}(\Omega)$. Moreover, $A f=a(x) \mathscr{A} f$ whenever $f \in H^{4}\left(\Omega, \Gamma_{1}\right)$ and that $A^{-1} g=(a(x) \mathscr{A})^{-1} g$ for any $g \in L^{2}(\Omega)$. Hence $A$ is an extension of $a(x) \mathscr{A}$ to the space $H_{0}^{2}(\Omega)$.

Just as in [15], it can be shown that $D\left(A^{1 / 2}\right)=L^{2}(\Omega)$ and $A^{1 / 2}$ is an isomorphism from $L^{2}(\Omega)$ onto $H$. Taking $s=\frac{3}{2}$ in (4.3) of Lemma 4.1 in Section 4, we can define the map $\Upsilon \in \mathcal{L}\left(L^{2}(\Gamma), H^{3 / 2}(\Omega)\right)$ such that $\Upsilon u=v$ if and only if

$$
\left\{\begin{array}{l}
a(x) \mathcal{A}^{2} v=0 \text { in } \Omega \\
v_{1}=v_{2}, \frac{\partial v_{1}}{\partial \nu_{\mathcal{A}}}=\frac{\partial v_{2}}{\partial \nu_{\mathcal{A}}}, a_{1} \mathcal{A} v_{1}=a_{2} \mathcal{A} v_{2}, a_{1} \frac{\partial \mathcal{A} v_{1}}{\partial \nu_{\mathcal{A}}}=a_{2} \frac{\partial \mathcal{A} v_{2}}{\partial \nu_{\mathcal{A}}} \text { on } \Gamma_{1}, \\
v_{2}=0, \frac{\partial v_{2}}{\partial \nu_{\mathcal{A}}}=u \text { on } \Gamma .
\end{array}\right.
$$

Since $D(A)$ is dense in $H$, so is $D\left(A^{1 / 2}\right)$. We identify $H$ with its dual $H^{\prime}$. Then the following relations hold:

$$
D\left(A^{1 / 2}\right) \hookrightarrow H=H^{\prime} \hookrightarrow\left(D\left(A^{1 / 2}\right)\right)^{\prime} .
$$

An extension $\tilde{A} \in \mathcal{L}\left(D(A)^{1 / 2},\left(D\left(A^{1 / 2}\right)\right)^{\prime}\right)$ of $A$ is defined by

$$
\langle\tilde{A} f, g\rangle_{\left(D\left(A^{1 / 2}\right)\right)^{\prime} \times D\left(A^{1 / 2}\right)}=\left\langle A^{1 / 2} f, A^{1 / 2} g\right\rangle_{H}, \forall f, g \in D\left(A^{1 / 2}\right) .
$$

So $(1.1)$ can be written in $\left(D\left(A^{1 / 2}\right)\right)^{\prime}$ as

$$
\ddot{w}+\tilde{A} w=B u
$$

where $B \in \mathcal{L}\left(U,\left(D\left(A^{1 / 2}\right)\right)^{\prime}\right)$ is given by

$$
B u=\tilde{A} \Upsilon u, \forall u \in U .
$$

Define $B^{*} \in \mathcal{L}\left(D\left(A^{1 / 2}\right), U\right)$ by

$$
\left\langle B^{*} f, u\right\rangle_{U}=\langle f, B u\rangle_{D\left(A^{1 / 2}\right) \times\left(D\left(A^{1 / 2}\right)\right)^{\prime}}, \forall f \in D\left(A^{1 / 2}\right), u \in U .
$$

Then for any $f \in D\left(A^{1 / 2}\right)$ and $u \in C_{0}^{\infty}\left(\Gamma_{0}\right)$, we have

$$
\begin{aligned}
\langle f, B u\rangle_{D\left(A^{1 / 2}\right) \times\left(D\left(A^{1 / 2}\right)\right)^{\prime}} & =\left\langle f, \tilde{A} \tilde{A}^{-1} B u\right\rangle_{D\left(A^{1 / 2}\right) \times\left(D\left(A^{1 / 2}\right)\right)^{\prime}}=\left\langle A^{1 / 2} f, A^{1 / 2} \tilde{A}^{-1} B u\right\rangle_{H} \\
& =\left\langle A^{1 / 2} f, A^{1 / 2} \Upsilon u\right\rangle_{H}=\langle f, \Upsilon u\rangle_{L^{2}(\Omega)} \\
& =\left\langle A A^{-1} f, \Upsilon u\right\rangle_{L^{2}(\Omega)}=\left\langle-\mathcal{A}\left(\mathscr{A}^{-1} f\right), u\right\rangle_{U} .
\end{aligned}
$$

In the last step, we used Green's second formula twice and the connection boundary conditions on $\Gamma_{1}$. Since $C_{0}^{\infty}(\Gamma)$ is dense in $L^{2}(\Gamma)$, we finally obtain

$$
B^{*} f=-\left.\mathcal{A}\left(\mathscr{A}^{-1} f\right)\right|_{\Gamma}, \forall f \in D\left(A^{1 / 2}\right)=L^{2}(\Omega) .
$$


Now we have formulated the open-loop system (1.1) into an abstract form of a secondorder collocated system in $\mathcal{H}$ :

$$
\left\{\begin{array}{l}
\ddot{w}(\cdot, t)+\tilde{A} w(\cdot, t)=B u(\cdot, t), \\
y(\cdot, t)=B^{*} \dot{w}(\cdot, t),
\end{array}\right.
$$

where $\tilde{A}, B$ and $B^{*}$ are defined by (2.3), (2.4) and (2.5), respectively. The system (2.6) was well studied in $[2,11]$.

To end this section, we list some basic facts in Riemannian geometry that we need in subsequent sections (see also [16]).

Let $\left(\mathbb{R}^{n}, g\right)$ be the Riemannian manifold defined in Section 1. For any $\varphi \in C^{2}\left(\mathbb{R}^{n}\right)$ and $N=\sum_{i=1}^{n} \gamma_{i}(x) \frac{\partial}{\partial x_{i}}$, denote

$$
\begin{aligned}
& \operatorname{div}_{0}(N):=\sum_{i=1}^{n} \frac{\partial \gamma_{i}(x)}{\partial x_{i}}, D \varphi:=\nabla_{g} \varphi=\sum_{i, j=1}^{n} \frac{\partial \varphi}{\partial x_{i}} a_{i j} \frac{\partial}{\partial x_{j}}, \\
& \operatorname{div}_{g}(N):=\sum_{i=1}^{n} \frac{1}{\sqrt{\mathcal{G}(x)}} \frac{\partial}{\partial x_{i}}\left(\sqrt{\mathcal{G}(x)} \gamma_{i}(x)\right), \\
& \Delta_{g} \varphi:=\sum_{i, j=1}^{n} \frac{1}{\sqrt{\mathcal{G}}} \frac{\partial}{\partial x_{i}}\left(\sqrt{\mathcal{G}} a_{i j} \frac{\partial \varphi}{\partial x_{j}}\right)=\mathcal{A} \varphi-(D f) \varphi, f(x)=\frac{1}{2} \log \operatorname{det}\left(a_{i j}(x)\right),
\end{aligned}
$$

where $\operatorname{div}_{0}$ is the divergence operator on Euclidean space $\mathbb{R}^{n}$ and $\nabla_{g}, \operatorname{div}_{g}$ and $\Delta_{g}$ are the gradient operator, the divergence operator and the Beltrami-Laplace operator on $\left(\mathbb{R}^{n}, g\right)$ respectively.

Let $\mu=\frac{\nu_{\mathcal{A}}}{\left|\nu_{\mathcal{A}}\right|_{g}} \cdot \mu$ is the unit outward-pointing normal to $\partial \Omega$ in terms of the Riemannian metric $g$. The following Lemma 2.1 provides some useful identities ([28], pp. 128, 138).

Lemma 2.1. Let $\varphi, \psi \in C^{1}(\bar{\Omega})$ and let $N$ be a vector field on $\left(\mathbb{R}^{n}, g\right)$. Then we have

(1) divergence formula and theorem:

$$
\begin{gathered}
\operatorname{div}_{0}(\varphi N)=\varphi \operatorname{div}_{0}(N)+N(\varphi), \quad \operatorname{div}_{g}(\varphi N)=\varphi \operatorname{div}_{g}(N)+N(\varphi), \\
\int_{\Omega} \operatorname{div}_{0}(N) d x=\int_{\partial \Omega} N \cdot \nu d \Gamma, \quad \int_{\Omega} \operatorname{div}_{g}(N) d x=\int_{\partial \Omega}\langle N, \mu\rangle_{g} d \Gamma,
\end{gathered}
$$

(2) Green's identities:

$$
\begin{aligned}
& \int_{\Omega} \psi \mathcal{A} \varphi d x=\int_{\partial \Omega} \psi \frac{\partial \varphi}{\partial \nu_{\mathcal{A}}} d \Gamma-\int_{\Omega}\left\langle\nabla_{g} \varphi, \nabla_{g} \psi\right\rangle_{g} d x \\
& \int_{\Omega} \psi \Delta_{g} \varphi d \Omega=\int_{\partial \Omega} \psi \frac{\partial \varphi}{\partial \mu} d S-\int_{\Omega}\left\langle\nabla_{g} \varphi, \nabla_{g} \psi\right\rangle_{g} d \Omega
\end{aligned}
$$

where $d \Omega$ and $d S$ are the volume elements of $\Omega$ and $\partial \Omega$, respectively.

Denote by $T^{2}\left(\mathbb{R}_{x}^{n}\right)$ the set of all covariant tensors of order 2 on $\mathbb{R}_{x}^{n}$. Then $T^{2}\left(\mathbb{R}_{x}^{n}\right)$ is an inner product space of dimension $n^{2}$ with inner product of the following:

$$
\langle F, G\rangle_{T^{2}\left(\mathbb{R}_{x}^{n}\right)}=\sum_{i, j=1}^{n} F\left(e_{i}, e_{j}\right) G\left(e_{i}, e_{j}\right), \forall F, G \in T^{2}\left(\mathbb{R}_{x}^{n}\right),
$$


where $\left\{e_{i}\right\}_{i=1}^{n}$ is an arbitrarily chosen orthonormal basis of $\left(\mathbb{R}_{x}^{n}, g\right)$.

Let $\mathfrak{X}\left(\mathbb{R}^{n}\right)$ be the set of all tangential vector fields on $\mathbb{R}^{n}$. Denote by $\triangle: \mathfrak{X}\left(\mathbb{R}^{n}\right) \rightarrow$ $\mathfrak{X}\left(\mathbb{R}^{n}\right)$ the Hodge-Laplace operator acting on the tangential fields; then for any orthonormal basis $\left\{e_{i}\right\}_{i=1}^{n}$ and its dual basis $\left\{w^{i}\right\}_{i=1}^{n}$ at $\left(\mathbb{R}_{x}^{n}, g\right)$, we have the following Weitzenböck formula (see $[32])$ :

$$
\triangle=-\sum_{k=1}^{n} D_{e_{k} e_{k}}^{2}+\sum_{j, k=1}^{n} e^{k} \wedge i\left(w^{j}\right) R\left(e_{k}, e_{j}\right),
$$

where for any $X, Y \in \mathfrak{X}\left(\mathbb{R}^{n}\right)$ and $\eta \in A\left(\mathbb{R}^{n}\right)$, the set of all the differential forms on $\mathbb{R}^{n}$, $D_{X Y}^{2}:=D_{X} D_{Y}-D_{D_{X} Y}$ is a second order covariant differential operator, $R(X, Y)=$ $-D_{X} D_{Y}+D_{Y} D_{X}+D_{[X, Y]}$ is the curvature operator with regard to the metric $g$, and $i(\eta) X:=\eta(X)$ is the interior product operator. Furthermore, it has ([34]):

$$
\begin{gathered}
\Delta_{g}(N(\varphi))=(-\triangle N)(\varphi)+2\left\langle D N, D^{2} \varphi\right\rangle_{T^{2}\left(\mathbb{R}_{x}^{n}\right)}+N\left(\Delta_{g} \varphi\right)+\operatorname{Ric}(N, D \varphi), \\
N\left(\Delta_{g} \varphi\right)=N(\mathcal{A} \varphi)-D^{2} f(N, D \varphi)-D^{2} \varphi(N, D f), \forall \varphi \in C^{2}\left(\mathbb{R}^{n}\right),
\end{gathered}
$$

where $f(x)=\frac{1}{2} \log \operatorname{det}\left(a_{i j}(x)\right)$ and $\operatorname{Ric}(\cdot, \cdot)$ is the Ricci curvature tensor with respect to the Riemannian metric $g, D^{2} f$ and $D^{2} \varphi$ are the Hessians of $f$ and $\varphi$ in terms of $g$, respectively. The identity (2.9) is actually proved by (5.10) in Lemma 5.1 of Section 5 , while the proof of (2.8) is presented in the Appendix.

The following Lemma 2.2 is straightforward.

Lemma 2.2. Let $\varphi \in C^{2}(\bar{\Omega})$. Then there is a constant $C$ depending on $g, N$ and $\Omega$ only such that

(1)

$$
\begin{aligned}
& \sup _{x \in \bar{\Omega}}\left|\mathcal{A}\left(\operatorname{div}_{g}(N)\right)\right| \leq C, \quad \sup _{x \in \bar{\Omega}}\left|D f\left(\operatorname{div}_{g}(N)\right)\right| \leq C, \quad \sup _{x \in \bar{\Omega}}\left|\operatorname{div}_{0}(D f)\right| \leq C, \\
& \sup _{x \in \bar{\Omega}}\left|\operatorname{div}_{0}(N)\right| \leq C, \quad \sup _{x \in \bar{\Omega}}\left|\operatorname{div}_{g}(N)\right| \leq C, \quad \sup _{x \in \partial \Omega}\left|\frac{1}{\left|\nu_{\mathcal{A}}\right|_{g}}\right| \leq C, \quad \sup _{x \in \partial \Omega}|D f \cdot \nu| \leq C .
\end{aligned}
$$

$(2)$

$$
\begin{aligned}
& \left|\left\langle D \varphi, D\left(\operatorname{div}_{g}(N)\right)\right\rangle_{g}\right| \leq|D \varphi|_{g}\left|D\left(\operatorname{div}_{g}(N)\right)\right|_{g} \leq C|D \varphi|_{g}, \\
& |(\triangle N) \varphi|_{g} \leq C|\triangle N|_{g}|D \varphi|_{g} \leq C|D \varphi|_{g}, \\
& \left|\left\langle D N, D^{2} \varphi\right\rangle_{T^{2}\left(\mathbb{R}_{x}^{n}\right)}\right| \leq C|D N|_{g}\left|D^{2} \varphi\right|_{g} \leq C\left|D^{2} \varphi\right|_{g}, \\
& \left|D^{2} f(N, D \varphi)\right| \leq\left|D^{2} f\right|_{g}|N|_{g}|D \varphi|_{g} \leq C|D \varphi|_{g}, \\
& \left|D^{2} \varphi(N, D f)\right| \leq\left|D^{2} \varphi\right|_{g}|N|_{g}|D f|_{g} \leq C\left|D^{2} \varphi\right|_{g}, \\
& |\operatorname{Ric}(N, D \varphi)| \leq|\operatorname{Ric}|_{g}|N|_{g}|D \varphi|_{g} \leq C|D \varphi|_{g}, \quad|D f(\varphi)| \leq C|D \varphi|_{g}, \\
& |N(\varphi)| \leq C|D \varphi|_{g}, \quad|D f(N(\varphi))| \leq C\left|D^{2} \varphi\right|_{g}, \quad|\mathcal{A} \varphi| \leq C\left|D^{2} \varphi\right|_{g} .
\end{aligned}
$$

$$
\int_{\Omega}|\varphi|^{2} d x \leq C\|\varphi\|_{H^{2}(\Omega)}^{2}, \int_{\Omega}|D \varphi|_{g}^{2} d x \leq C\|\varphi\|_{H^{2}(\Omega)}^{2}, \int_{\Omega}\left|D^{2} \varphi\right|_{g}^{2} d x \leq C\|\varphi\|_{H^{2}(\Omega)}^{2} .
$$


3. The proof of Theorem 1.1. In this section, we use $C_{T}$ to denote some positive constant that is independent of $(y, u)$ although it may change values in different contexts. We rewrite (1.1) with zero initial values as the following equation (3.1):

$$
\left\{\begin{array}{l}
\ddot{w}(x, t)+a(x) \mathcal{A}^{2} w(x, t)=0,(x, t) \in Q \\
w(x, 0)=0, \dot{w}(x, 0)=0, x \in \Omega \\
w_{2}(x, t)=0, \frac{\partial w_{2}}{\partial \nu_{\mathcal{A}}}(x, t)=u(x, t),(x, t) \in \Sigma \\
w_{1}(x, t)=w_{2}(x, t), \frac{\partial w_{1}}{\partial \nu_{\mathcal{A}}}(x, t)=\frac{\partial w_{2}}{\partial \nu_{\mathcal{A}}}(x, t),(x, t) \in \Sigma_{1}, \\
a_{1} \mathcal{A} w_{1}(x, t)=a_{2} \mathcal{A} w_{2}(x, t), a_{1} \frac{\partial \mathcal{A} w_{1}}{\partial \nu_{\mathcal{A}}}(x, t)=a_{2} \frac{\partial \mathcal{A} w_{2}}{\partial \nu_{\mathcal{A}}}(x, t),(x, t) \in \Sigma_{1}, \\
y(x, t)=-\mathcal{A}\left(\mathscr{A}^{-1} \dot{w}(x, t)\right),(x, t) \in \Sigma
\end{array}\right.
$$

By [31] (see also Proposition A1 of [19]), Theorem 1.1 is equivalent to saying that the solution of (3.1) satisfies

$$
\|y\|_{L^{2}(0, T ; U)}^{2} \leq C_{T}\|u\|_{L^{2}(0, T ; U)}^{2}, \forall u \in L^{2}(0, T ; U) .
$$

We return to the smoother space $H_{0}^{2}(\Omega)$ by the following transformation:

$$
z=A^{-1} \dot{w} \text {. }
$$

Then $z$ satisfies

$$
\left\{\begin{array}{l}
\ddot{z}(x, t)+a(x) \mathcal{A}^{2} z(x, t)=\Upsilon u_{t}(x, t),(x, t) \in Q \\
z(x, 0)=z^{0}(x), \dot{z}(x, 0)=z^{1}(x), x \in \Omega \\
z_{2}(x, t)=0, \frac{\partial z_{2}}{\partial \nu_{\mathcal{A}}}(x, t)=0,(x, t) \in \Sigma \\
z_{1}(x, t)=z_{2}(x, t), \frac{\partial z_{1}}{\partial \nu_{\mathcal{A}}}(x, t)=\frac{\partial z_{2}}{\partial \nu_{\mathcal{A}}}(x, t),(x, t) \in \Sigma_{1}, \\
a_{1} \mathcal{A} z_{1}(x, t)=a_{2} \mathcal{A} z_{2}(x, t), a_{1} \frac{\partial \mathcal{A} z_{1}}{\partial \nu_{\mathcal{A}}}(x, t)=a_{2} \frac{\partial \mathcal{A} z_{2}}{\partial \nu_{\mathcal{A}}}(x, t),(x, t) \in \Sigma_{1}
\end{array}\right.
$$

By (2.5), the output becomes

$$
y(x, t)=-\left.\mathcal{A} z_{2}(x, t)\right|_{\Sigma} .
$$

Therefore, Theorem 1.1 is valid if and only if for some (and hence for all) $T>0$, there exists a $C_{T}>0$ such that the solution of (3.2) satisfies

$$
\int_{\Sigma}|\mathcal{A} z(x, t)|^{2} d \Sigma \leq C_{T} \int_{\Sigma}|u(x, t)|^{2} d \Sigma
$$

Proof. We split the proof into eight steps.

Step 1. Let $N$ be a vector field of class $C^{2}$ on $\bar{\Omega}$ such that (Lemma 4.1, [15])

$$
N(x)=\mu(x), x \in \Gamma ;|N|_{g} \leq 1, \quad x \in \Omega .
$$


Multiply both sides of the first equation of $(3.2)$ by $N(\bar{z})$ and integrate over $[0, T] \times \Omega$, to give

$$
\int_{0}^{T} \int_{\Omega} \ddot{z} N(\bar{z}) d x d t+\int_{0}^{T} \int_{\Omega} a(x) \mathcal{A}^{2} z N(\bar{z}) d x d t-\int_{0}^{T} \int_{\Omega} \Upsilon u_{t} N(\bar{z}) d x d t=0
$$

Compute the first term on the left hand side of (3.5) to yield

$$
\begin{aligned}
& \int_{0}^{T} \int_{\Omega} \ddot{z} N(\bar{z}) d x d t=\left.\int_{\Omega} \dot{z} N(\bar{z}) d x\right|_{0} ^{T}-\int_{0}^{T} \int_{\Omega} \dot{z} N(\overline{\dot{z}}) d x d t \\
& =\left.\int_{\Omega} \dot{z} N(\bar{z}) d x\right|_{0} ^{T}-\left.\int_{\Omega} z N(\overline{\dot{z}}) d x\right|_{0} ^{T}+\int_{0}^{T} \int_{\Omega} z N(\overline{\ddot{z}}) d x d t \\
& =\left.\int_{\Omega} \dot{z} N(\bar{z}) d x\right|_{0} ^{T}-\left.\int_{\Omega}\left[\operatorname{div}_{g}(z \overline{\dot{z}} N)-\overline{\dot{z}} z \operatorname{div}_{g}(N)-\overline{\dot{z}} N(z)\right] d x\right|_{0} ^{T} \\
& +\int_{0}^{T} \int_{\Omega}\left[\operatorname{div}_{g}(z \overline{\ddot{z}} N)-\overline{\ddot{z}} z \operatorname{div}_{g}(N)-\overline{\ddot{z}} N(z)\right] d x d t \\
& =\left.2 \operatorname{Re} \int_{\Omega} \dot{z} N(\bar{z}) d x\right|_{0} ^{T}+\left.\int_{\Omega} \overline{\dot{z}} z \operatorname{div}_{g}(N) d x\right|_{0} ^{T} \\
& \quad+\int_{0}^{T} \int_{\Omega}\left[z a(x) \mathcal{A}^{2} \bar{z} \operatorname{div}_{g}(N)-\Upsilon \overline{u_{t}} z \operatorname{div}_{g}(N)-\overline{\ddot{z}} N(z)\right] d x d t .
\end{aligned}
$$

Hence

$$
\begin{array}{r}
\operatorname{Re} \int_{0}^{T} \int_{\Omega} \ddot{z} N(\bar{z}) d x d t=\left.\operatorname{Re} \int_{\Omega} \dot{z} N(\bar{z}) d x\right|_{0} ^{T}+\left.\frac{1}{2} \int_{\Omega} \dot{z} z \operatorname{div}_{g}(N) d x\right|_{0} ^{T} \\
\quad-\frac{1}{2} \int_{0}^{T} \int_{\Omega} \Upsilon \overline{u_{t}} z \operatorname{div}_{g}(N) d x d t+\frac{1}{2} \int_{0}^{T} \int_{\Omega} z a(x) \mathcal{A}^{2} \bar{z} \operatorname{div}_{g}(N) d x d t .
\end{array}
$$

By Green's second formula in the Riemannian manifold and the fact that

$$
\frac{\partial z}{\partial \mu}=\left\langle\nabla_{g} z, \mu\right\rangle_{g}, \quad \frac{\partial}{\partial \mu}=\mu=\frac{1}{\left|\nu_{\mathcal{A}}\right|_{g}} \frac{\partial}{\partial \nu_{\mathcal{A}}} \text { on } \Gamma \text { and } \Gamma_{1}
$$


the last term of (3.6), by taking the boundary conditions into account, is further expressed as

$$
\begin{aligned}
& \frac{1}{2} \int_{0}^{T} \int_{\Omega} z a(x) \mathcal{A}^{2} \bar{z} \operatorname{div}_{g}(N) d x d t=\frac{1}{2} \int_{0}^{T} \int_{\Omega} z a(x)\left[\left(\Delta_{g}+D f\right)(\mathcal{A} \bar{z})\right] \operatorname{div}_{g}(N) d x d t \\
= & \frac{1}{2} \int_{0}^{T} \int_{\Omega} z a(x) \Delta_{g}(\mathcal{A} \bar{z}) \operatorname{div}_{g}(N) d x d t+\frac{1}{2} \int_{0}^{T} \int_{\Omega} z a(x) D f(\mathcal{A} \bar{z}) \operatorname{div}_{g}(N) d x d t \\
= & \frac{1}{2} \int_{0}^{T} \int_{\Omega} a(x) \mathcal{A} \bar{z} \Delta_{g}\left(z \operatorname{div}_{g}(N)\right) d x d t+\frac{1}{2} \int_{0}^{T} \int_{\Gamma} a_{2} z_{2} \operatorname{div}_{g}(N) \frac{\partial\left(\mathcal{A} \bar{z}_{2}\right)}{\partial \mu} d \Gamma d t \\
& +\frac{1}{2} \int_{0}^{T} \int_{\Gamma_{1}} a_{2} z_{2} \operatorname{div}_{g}(N) \frac{\partial\left(\mathcal{A} \bar{z}_{2}\right)}{\partial \mu} d \Gamma d t-\frac{1}{2} \int_{0}^{T} \int_{\Gamma_{1}} a_{1} z_{1} \operatorname{div}_{g}(N) \frac{\partial\left(\mathcal{A} \bar{z}_{1}\right)}{\partial \mu} d \Gamma d t \\
& -\frac{1}{2} \int_{0}^{T} \int_{\Gamma} a_{2} \mathcal{A} \bar{z}_{2} \frac{\partial\left(z_{2} \operatorname{div}_{g}(N)\right)}{\partial \mu} d \Gamma d t-\frac{1}{2} \int_{0}^{T} \int_{\Gamma_{1}} a_{2} \mathcal{A} \bar{z}_{2} \frac{\partial\left(z_{2} \operatorname{div}{ }_{g}(N)\right)}{\partial \mu} d \Gamma d t \\
& +\frac{1}{2} \int_{0}^{T} \int_{\Gamma_{1}} a_{1} \mathcal{A} \bar{z}_{1} \frac{\partial\left(z_{1} \operatorname{div}_{g}(N)\right)}{\partial \mu} d \Gamma d t+\frac{1}{2} \int_{0}^{T} \int_{\Omega} z a(x) D f(\mathcal{A} \bar{z}) \operatorname{div}_{g}(N) d x d t \\
= & \frac{1}{2} \int_{0}^{T} \int_{\Omega} a(x) \mathcal{A} \bar{z}\left[\mathcal{A} z \operatorname{div}_{g}(N)+2\left\langle D z, D\left(\operatorname{div}_{g}(N)\right)\right\rangle_{g}+z \mathcal{A}\left(\operatorname{div}_{g}(N)\right)\right] d x d t \\
& -\frac{1}{2} \int_{0}^{T} \int_{\Omega} a(x) \mathcal{A} \bar{z} D f\left(z \operatorname{div}_{g}(N)\right) d x d t+\frac{1}{2} \int_{0}^{T} \int_{\Omega} z a(x) D f(\mathcal{A} \bar{z}) \operatorname{div}_{g}(N) d x d t .
\end{aligned}
$$

Substitute (3.8) into (3.6) to obtain

$$
\begin{aligned}
\operatorname{Re} & \int_{0}^{T} \int_{\Omega} \ddot{z} N(\bar{z}) d x d t=\left.\operatorname{Re} \int_{\Omega} \dot{z} N(\bar{z}) d x\right|_{0} ^{T}+\left.\frac{1}{2} \int_{\Omega} \overline{\dot{z}} z \operatorname{div}_{g}(N) d x\right|_{0} ^{T} \\
& -\frac{1}{2} \int_{0}^{T} \int_{\Omega} \Upsilon \overline{u_{t}} z \operatorname{div}_{g}(N) d x d t+\frac{1}{2} \int_{0}^{T} \int_{\Omega} a(x)|\mathcal{A} z|^{2} \operatorname{div}_{g}(N) d x d t \\
& +\int_{0}^{T} \int_{\Omega} a(x) \mathcal{A} \bar{z}\left\langle D z, D\left(\operatorname{div}_{g}(N)\right)\right\rangle_{g} d x d t+\frac{1}{2} \int_{0}^{T} \int_{\Omega} a(x) z \mathcal{A} \bar{z} \mathcal{A}\left(\operatorname{div}_{g}(N)\right) d x d t \\
& -\frac{1}{2} \int_{0}^{T} \int_{\Omega} a(x) \mathcal{A} \bar{z} D f\left(z \operatorname{div}_{g}(N)\right) d x d t+\frac{1}{2} \int_{0}^{T} \int_{\Omega} z a(x) D f(\mathcal{A} \bar{z}) \operatorname{div}_{g}(N) d x d t
\end{aligned}
$$

Next, by Lemma 2.1 of [34] and (3.7),

$$
\left\{\begin{array}{l}
z_{1}-z_{2}=\frac{\partial\left(z_{1}-z_{2}\right)}{\partial \nu_{\mathcal{A}}}=0 \Rightarrow H\left(z_{1}-z_{2}\right)=0 \text { for any vector field } H \text { on } \Gamma_{1} \\
\frac{\partial^{2}\left(z_{1}-z_{2}\right)}{\partial \mu^{2}}=\frac{1}{\left|\nu_{\mathcal{A}}\right|_{g}^{2}} \frac{\partial^{2}\left(z_{1}-z_{2}\right)}{\partial \nu_{\mathcal{A}}^{2}}=\frac{1}{\left|\nu_{\mathcal{A}}\right|_{g}^{2}}\left(\nu_{\mathcal{A}} \cdot \nu\right) \mathcal{A}\left(z_{1}-z_{2}\right)=\Delta_{g}\left(z_{1}-z_{2}\right) .
\end{array}\right.
$$

Applying Green's second formula in the Riemannian manifold again, and taking (2.8), (2.9), (3.7), (3.10) and boundary conditions into account, the second term on the left 
hand side of (3.5) is computed as

$$
\begin{aligned}
& \int_{0}^{T} \int_{\Omega} a(x) \mathcal{A}^{2} z N(\bar{z}) d x d t=\int_{0}^{T} \int_{\Omega} a(x)\left[\left(\Delta_{g}+D f\right)(\mathcal{A} z)\right] N(\bar{z}) d x d t \\
& =\int_{0}^{T} \int_{\Omega} a(x) \Delta_{g}(\mathcal{A} z) N(\bar{z}) d x d t+\int_{0}^{T} \int_{\Omega} a(x) D f(\mathcal{A} z) N(\bar{z}) d x d t \\
& =\int_{0}^{T} \int_{\Omega} a(x) \mathcal{A} z \Delta_{g}(N(\bar{z})) d x d t+\int_{0}^{T} \int_{\Gamma} N\left(\bar{z}_{2}\right) a_{2} \frac{\partial\left(\mathcal{A} z_{2}\right)}{\partial \mu} d \Gamma d t \\
& +\int_{0}^{T} \int_{\Gamma_{1}} N\left(\bar{z}_{2}\right) a_{2} \frac{\partial\left(\mathcal{A} z_{2}\right)}{\partial \mu} d \Gamma d t-\int_{0}^{T} \int_{\Gamma_{1}} N\left(\bar{z}_{1}\right) a_{1} \frac{\partial\left(\mathcal{A} z_{1}\right)}{\partial \mu} d \Gamma d t \\
& -\int_{0}^{T} \int_{\Gamma} a_{2} \mathcal{A} z_{2} \frac{\partial\left(N\left(\bar{z}_{2}\right)\right)}{\partial \mu} d \Gamma d t-\int_{0}^{T} \int_{\Gamma_{1}} a_{2} \mathcal{A} z_{2} \frac{\partial\left(N\left(\bar{z}_{2}\right)\right)}{\partial \mu} d \Gamma d t \\
& +\int_{0}^{T} \int_{\Gamma_{1}} a_{1} \mathcal{A} z_{1} \frac{\partial\left(N\left(\bar{z}_{1}\right)\right)}{\partial \mu} d \Gamma d t+\int_{0}^{T} \int_{\Omega} a(x) D f(\mathcal{A} z) N(\bar{z}) d x d t \\
& =\int_{0}^{T} \int_{\Omega} a(x) \mathcal{A} z \Delta_{g}(N(\bar{z})) d x d t-\int_{0}^{T} \int_{\Gamma} a_{2} \mathcal{A} z_{2} \frac{\partial^{2} \bar{z}_{2}}{\partial \mu^{2}} d \Gamma d t-\int_{0}^{T} \int_{\Gamma_{1}} a_{2} \mathcal{A} z_{2} \frac{\partial^{2} \bar{z}_{2}}{\partial \mu^{2}} d \Gamma d t \\
& +\int_{0}^{T} \int_{\Gamma_{1}} a_{1} \mathcal{A} z_{1} \frac{\partial^{2} \bar{z}_{1}}{\partial \mu^{2}} d \Gamma d t+\int_{0}^{T} \int_{\Omega} a(x) D f(\mathcal{A} z) N(\bar{z}) d x d t \\
& =\int_{0}^{T} \int_{\Omega} a(x) \mathcal{A} z\left[(-\triangle N)(\bar{z})+2\left\langle D N, D^{2} \bar{z}\right\rangle_{T^{2}\left(\mathbb{R}_{x}^{n}\right)}+N\left(\Delta_{g} \bar{z}\right)\right. \\
& +\operatorname{Ric}(N, D \bar{z})] d x d t-\int_{0}^{T} \int_{\Gamma} a_{2}\left|\mathcal{A} z_{2}\right|^{2} d \Gamma d t \\
& +\int_{0}^{T} \int_{\Gamma_{1}}\left(a_{1}\left|\mathcal{A} z_{1}\right|^{2}-a_{2}\left|\mathcal{A} z_{2}\right|^{2}\right) d \Gamma d t+\int_{0}^{T} \int_{\Omega} a(x) D f(\mathcal{A} z) N(\bar{z}) d x d t \\
& =\int_{0}^{T} \int_{\Omega} a(x) \mathcal{A} z\left[(-\triangle N)(\bar{z})+2\left\langle D N, D^{2} \bar{z}\right\rangle_{T^{2}\left(\mathbb{R}_{x}^{n}\right)}+N(\mathcal{A} \bar{z})-D^{2} f(N, D \bar{z})\right. \\
& \left.-D^{2} \bar{z}(N, D f)+\operatorname{Ric}(N, D \bar{z})\right] d x d t+\int_{0}^{T} \int_{\Omega} a(x) D f(\mathcal{A} z) N(\bar{z}) d x d t \\
& -\int_{0}^{T} \int_{\Gamma} a_{2}\left|\mathcal{A} z_{2}\right|^{2} d \Gamma d t+\int_{0}^{T} \int_{\Gamma_{1}} \frac{\left(a_{2}-a_{1}\right)}{a_{1} a_{2}}\left|a_{1} \mathcal{A} z_{1}\right|^{2} d \Gamma d t .
\end{aligned}
$$

In the last step of (3.11), the identity $\frac{\partial^{2} \overline{z_{2}}}{\partial \mu^{2}}=\Delta_{g} \bar{z}_{2}=\mathcal{A} \bar{z}_{2}$ on $\Gamma$ that is similar to (3.10) was used. 
Furthermore, by the divergence formula, one has

$$
\begin{aligned}
& \operatorname{Re} \int_{0}^{T} \int_{\Omega} a(x) \mathcal{A} z N(\mathcal{A} \bar{z}) d x d t=\frac{1}{2} \int_{0}^{T} \int_{\Omega} N\left(a(x)|\mathcal{A} z|^{2}\right) d x d t \\
= & \frac{1}{2} \int_{0}^{T} \int_{\Gamma} a_{2}\left|\mathcal{A} z_{2}\right|^{2} d \Gamma d t+\frac{1}{2} \int_{0}^{T} \int_{\Gamma_{1}} a_{2}\left|\mathcal{A} z_{2}\right|^{2} d \Gamma d t-\frac{1}{2} \int_{0}^{T} \int_{\Gamma_{1}} a_{1}\left|\mathcal{A} z_{1}\right|^{2} d \Gamma d t \\
& -\frac{1}{2} \int_{0}^{T} \int_{\Omega} a(x)|\mathcal{A} z|^{2} \operatorname{div}_{g}(N) d x d t \\
= & \frac{1}{2} \int_{0}^{T} \int_{\Gamma} a_{2}\left|\mathcal{A} z_{2}\right|^{2} d \Gamma d t-\frac{1}{2} \int_{0}^{T} \int_{\Gamma_{1}} \frac{\left(a_{2}-a_{1}\right)}{a_{1} a_{2}}\left|a_{1} \mathcal{A} z_{1}\right|^{2} d \Gamma d t \\
& -\frac{1}{2} \int_{0}^{T} \int_{\Omega} a(x)|\mathcal{A} z|^{2} \operatorname{div}_{g}(N) d x d t .
\end{aligned}
$$

So (3.11) can be further expressed as

$$
\begin{aligned}
& \operatorname{Re} \int_{0}^{T} \int_{\Omega} a(x) \mathcal{A}^{2} z N(\bar{z}) d x d t \\
= & -\frac{1}{2} \int_{0}^{T} \int_{\Omega} a(x)|\mathcal{A} z|^{2} \operatorname{div}_{g}(N) d x d t+\operatorname{Re} \int_{0}^{T} \int_{\Omega} a(x) \mathcal{A} z[(-\triangle N)(z) \\
& \left.+2\left\langle D N, D^{2} \bar{z}\right\rangle_{T^{2}\left(\mathbb{R}_{x}^{n}\right)}-D^{2} f(N, D \bar{z})-D^{2} \bar{z}(N, D f)+\operatorname{Ric}(N, D \bar{z})\right] d x d t \\
& -\frac{1}{2} \int_{0}^{T} \int_{\Gamma} a_{2}\left|\mathcal{A} z_{2}\right|^{2} d \Gamma d t+\frac{1}{2} \int_{0}^{T} \int_{\Gamma_{1}} \frac{\left(a_{2}-a_{1}\right)}{a_{1} a_{2}}\left|a_{1} \mathcal{A} z_{1}\right|^{2} d \Gamma d t \\
& +\operatorname{Re} \int_{0}^{T} \int_{\Omega} a(x) D f(\mathcal{A} z) N(\bar{z}) d x d t .
\end{aligned}
$$

Finally, substitute (3.9) and (3.12) into (3.5) to obtain

$$
\begin{aligned}
& \frac{1}{2} \int_{0}^{T} \int_{\Gamma} a_{2}|\mathcal{A} z|^{2} d \Gamma d t+\frac{1}{2} \int_{0}^{T} \int_{\Gamma_{1}} \frac{\left(a_{1}-a_{2}\right)}{a_{1} a_{2}}\left|a_{1} \mathcal{A} z_{1}\right|^{2} d \Gamma d t \\
= & \mathrm{RHS}_{1}+\mathrm{RHS}_{2}+\mathrm{RHS}_{3}+\mathrm{b}_{0, \mathrm{~T}},
\end{aligned}
$$


where

$$
\begin{aligned}
\operatorname{RHS}_{1}= & \int_{0}^{T} \int_{\Omega} a(x) \mathcal{A} \bar{z}\left\langle D z, D\left(\operatorname{div}_{g}(N)\right\rangle_{g} d x d t+\frac{1}{2} \int_{0}^{T} \int_{\Omega} z a(x) \mathcal{A} \bar{z} A\left(\operatorname{div}_{g}(N)\right) d x d t\right. \\
& +\operatorname{Re} \int_{0}^{T} \int_{\Omega} a(x) \mathcal{A} z\left[(-\triangle N)(\bar{z})+2\left\langle D N, D^{2} \bar{z}\right\rangle_{T^{2}\left(\mathbb{R}_{x}^{n}\right)}-D^{2} f(N, D \bar{z})\right. \\
& \left.-D^{2} \bar{z}(N, D f)+\operatorname{Ric}(N, D \bar{z})\right] d x d t, \\
\mathrm{RHS}_{2}= & -\frac{1}{2} \int_{0}^{T} \int_{\Omega} a(x) \mathcal{A} \bar{z} D f\left(z \operatorname{div}_{g}(N)\right) d x d t+\frac{1}{2} \int_{0}^{T} \int_{\Omega} z a(x) \operatorname{div}_{g}(N) D f(\mathcal{A} \bar{z}) d x d t \\
& +\operatorname{Re} \int_{0}^{T} \int_{\Omega} a(x) D f(\mathcal{A} z) N(\bar{z}) d x d t, \\
\operatorname{RHS}_{3}= & -\frac{1}{2} \int_{0}^{T} \int_{\Omega} \Upsilon \overline{u_{t}} z \operatorname{div}_{g}(N) d x d t-\operatorname{Re} \int_{0}^{T} \int_{\Omega} \Upsilon u_{t} N(\bar{z}) d x d t, \\
\mathrm{~b}_{0, \mathrm{~T}}=\quad & \left.\operatorname{Re} \int_{\Omega} \dot{z} N(\bar{z}) d x\right|_{0} ^{T}+\left.\frac{1}{2} \operatorname{Re} \int_{\Omega} \dot{\dot{z} z} z \operatorname{div}_{g}(N) d x\right|_{0} ^{T} \cdot
\end{aligned}
$$

By the assumption on $a(x)$, we have

$$
\frac{1}{2} \int_{0}^{T} \int_{\Gamma_{1}} \frac{\left(a_{1}-a_{2}\right)}{a_{1} a_{2}}\left|a_{1} \mathcal{A} z_{1}\right|^{2} d \Gamma d t \geq 0
$$

Therefore, we only need to estimate term by term for the right hand side of (3.13).

Step 2 (estimate FOR RHS 1 ). Let $\Upsilon \overline{u_{t}}=0$ in (3.13). We note that the transformation $z=A^{-1} \dot{w} \in H_{0}^{2}(\Omega)$ implies $\dot{z}=A^{-1} \ddot{w}=-w \in L^{2}(\Omega)$. Then Equation (3.2) associates with a $\mathrm{C}_{0}$-group solution in the space $H_{0}^{2}(\Omega) \times L^{2}(\Omega)$; that is to say, for any $\left(z^{0}, z^{1}\right) \in H_{0}^{2}(\Omega) \times L^{2}(\Omega)$, the corresponding solution to (3.2) satisfies $(z, \dot{z}) \in H_{0}^{2}(\Omega) \times L^{2}(\Omega)$ and depends continuously on $\left(z^{0}, z^{1}\right)$ :

$$
\frac{1}{2} \int_{0}^{T} \int_{\Gamma} a(x)\left|\mathcal{A} z_{2}\right|^{2} d \Gamma d t \leq C_{T}\left\|\left(z^{0}, z^{1}\right)\right\|_{H_{0}^{2}(\Omega) \times L^{2}(\Omega)}^{2} .
$$

This shows that $B^{*}$ is admissible, and so is $B([8])$. In other words,

$$
u \mapsto\{w, \dot{w}\} \text { is continuous from } L^{2}(\Sigma) \rightarrow C\left([0, T] ; L^{2}(\Omega) \times H^{-2}(\Omega)\right) .
$$

By $(3.14), z(t) \in C\left([0, T] ; H_{0}^{2}(\Omega)\right)$ that is continuous in $u \in L^{2}(\Sigma)$. Hence

$$
\mathrm{RHS}_{1} \leq C_{T}\|u\|_{L^{2}(\Sigma)}^{2}, \forall u \in L^{2}(\Sigma)
$$

where we used Lemma 2.2 .

STEP 3 (ESTIMATE FOR $\mathrm{RHS}_{2}$ ). By formulae

$$
\begin{aligned}
& \operatorname{div}_{0}\left(a(x) z \operatorname{div}_{g}(N) \mathcal{A} \bar{z} D f\right) \\
& =z a(x) \operatorname{div}_{g}(N) D f(\mathcal{A} \bar{z})+a(x) \mathcal{A} \bar{z} D f\left(z \operatorname{div}_{g}(N)\right)+z a(x) \operatorname{div}_{g}(N) \mathcal{A} \bar{z} \operatorname{div}_{0}(D f)
\end{aligned}
$$

and

$$
\operatorname{div}_{0}(a(x) N(\bar{z}) \mathcal{A} z D f)=a(x) D f(\mathcal{A} z) N(\bar{z})+a(x) \mathcal{A} z D f(N(\bar{z}))+a(x) N(\bar{z}) \mathcal{A} z \operatorname{div}_{0}(D f)
$$


we have

$$
\begin{aligned}
& \frac{1}{2} \int_{0}^{T} \int_{\Omega} z a(x) \operatorname{div}_{g}(N) D f(\mathcal{A} \bar{z}) d x d t \\
= & \frac{1}{2} \int_{0}^{T} \int_{\Gamma} a_{2} z_{2} \operatorname{div}_{g}(N) \mathcal{A} \bar{z}_{2} D f \cdot \nu d \Gamma d t \\
+ & \frac{1}{2} \int_{0}^{T} \int_{\Gamma_{1}} a_{2} z_{2} \operatorname{div}_{g}(N) \mathcal{A} \bar{z}_{2} D f \cdot \nu d \Gamma d t-\frac{1}{2} \int_{0}^{T} \int_{\Gamma_{1}} a_{1} z_{1} \operatorname{div}_{g}(N) \mathcal{A} \bar{z}_{1} D f \cdot \nu d \Gamma d t \\
& -\frac{1}{2} \int_{0}^{T} \int_{\Omega} a(x) \mathcal{A} \bar{z} D f\left(z \operatorname{div}_{g}(N)\right) d x d t-\frac{1}{2} \int_{0}^{T} \int_{\Omega} z a(x) \operatorname{div}_{g}(N) \mathcal{A} \bar{z} \operatorname{div}_{0}(D f) d x d t \\
= & -\frac{1}{2} \int_{0}^{T} \int_{\Omega} a(x) \mathcal{A} \bar{z} D f\left(z \operatorname{div}_{g}(N)\right) d x d t \\
= & -\frac{1}{2} \int_{0}^{T} \int_{\Omega} z a(x) \operatorname{div}_{g}(N) \mathcal{A} \bar{z} \operatorname{div}_{0}(D f) d x d t .
\end{aligned}
$$

Similarly,

$$
\begin{aligned}
\int_{0}^{T} & \int_{\Omega} a(x) D f(\mathcal{A} z) N(\bar{z}) d x d t=\int_{0}^{T} \int_{\Gamma} N\left(\bar{z}_{2}\right) a_{2} \mathcal{A} z_{2} D f \cdot \nu d \Gamma \\
- & \int_{0}^{T} \int_{\Omega} a(x) \mathcal{A} z D f(N(\bar{z})) d x d t-\int_{0}^{T} \int_{\Omega} a(x) N(\bar{z}) \mathcal{A} z \operatorname{div}_{0}(D f) d x d t .
\end{aligned}
$$

By boundary conditions we see that the first term on the right hand side of (3.17) equals zero. Substitute (3.16) and (3.17) into $\mathrm{RHS}_{2}$ to get

$$
\begin{aligned}
\mathrm{RHS}_{2}= & -\int_{0}^{T} \int_{\Omega} a(x) \mathcal{A} \bar{z} D f\left(z \operatorname{div}_{g}(N)\right) d x d t \\
& -\operatorname{Re} \int_{0}^{T} \int_{\Omega} a(x) \mathcal{A} z D f(N(\bar{z})) d x d t \\
& -\frac{1}{2} \int_{0}^{T} \int_{\Omega} z a(x) \operatorname{div}_{g}(N) \mathcal{A} \bar{z} \operatorname{div}_{0}(D f) d x d t \\
& -\operatorname{Re} \int_{0}^{T} \int_{\Omega} a(x) N(\bar{z}) \mathcal{A} z \operatorname{div}_{0}(D f) d x d t
\end{aligned}
$$

Thus, along the same lines as in Step 2, we get

$$
\mathrm{RHS}_{2} \leq C_{T}\|u\|_{L^{2}(\Sigma)}^{2}, \forall u \in L^{2}(\Sigma)
$$

where we used Lemma 2.2 again.

The following Steps 4-6 are very similar to that of [16] for the variable coefficients case without transmission, but for the sake of completeness, we list the sketch of the proof here.

SteP 4 (REgularity of $\dot{z}$ ). To handle $\mathrm{RHS}_{3}$, we need the regularity of $\dot{z}$.

$$
\dot{z}=A^{-1} \ddot{w}=A^{-1}(-A w+\tilde{A} \Upsilon u)=-w+\Upsilon u \in L^{2}(\Sigma) .
$$


Since both $w \in C\left([0, T] ; L^{2}(\Omega)\right)$ and $\Upsilon u \in L^{2}(\Sigma)$ depend continuously on $u \in L^{2}(\Sigma)$, it follows that

$$
\dot{z} \in L^{2}(\Sigma) \text { continuously in } u \in L^{2}(\Sigma) .
$$

Step 5 (estimates of $\mathrm{RHS}_{3}$ And $b_{0, T}$ FOR Smoother $u$ ). To estimate both $\mathrm{RHS}_{3}$ and $b_{0, T}$, confine $u$ within a smoother class that is dense in $L^{2}(\Sigma)$,

$$
u \in C^{1}([0, T] \times \Gamma), \quad u(\cdot, 0)=u(\cdot, T)=0 .
$$

We will show the following two estimates

$$
\mathrm{RHS}_{3} \leq C_{T}\|u\|_{L^{2}(\Sigma)}^{2}
$$

and

$$
\mathrm{b}_{0, T} \leq C_{T}\|u\|_{L^{2}(\Sigma)}^{2}
$$

for all $u$ in the class of (3.22). From now on, we assume that $z_{0}=z_{1}=0$ in (3.2).

SteP 6 (Proof of (3.24)). By the facts that $\dot{w} \in C\left([0, T] ; H^{-2}(\Omega)\right)$ continuously in $u \in L^{2}(\Sigma), A^{-1} \in \mathcal{L}\left(H^{-2}(\Omega), H_{0}^{2}(\Omega)\right)$ and $\dot{w}(\cdot, 0)=0$, we have

$$
z(\cdot, 0)=0, z(\cdot, T)=A^{-1} \dot{w} \in H_{0}^{2}(\Omega) \text { continuously in } u \in L^{2}(\Sigma) .
$$

Next by $(3.20),(3.22)$ and $w(\cdot, 0)=0$,

$$
\left\{\begin{array}{l}
\dot{z}(\cdot, 0)=-w(\cdot, 0)+\Upsilon u(\cdot, 0)=0, \\
\dot{z}(\cdot, T)=-w(\cdot, T) \in L^{2}(\Omega) \text { continuously in } u \in L^{2}(\Sigma),
\end{array}\right.
$$

where we used the regularity (3.14).

Using (3.14), (3.25) and (3.26), we readily obtain

$$
\mathrm{b}_{0, \mathrm{~T}}=\left.\operatorname{Re} \int_{\Omega} \dot{z} N(z) d x\right|_{0} ^{T}+\left.\operatorname{Re} \frac{1}{2} \int_{\Omega} \overline{\dot{z}} z \operatorname{div}_{g}(N) d x\right|_{0} ^{T} \leq C_{T}\|u\|_{L^{2}(\Sigma)}^{2} .
$$

SteP 7 (PROOF OF (3.23)). For the second term with $u$ in the class (3.22), we integrate by parts with respect to $t$ and make use of the divergence theorem again to obtain

$$
\begin{aligned}
& -\operatorname{Re} \int_{0}^{T} \int_{\Omega} \Upsilon u_{t} N(z) d x d t=-\left.\operatorname{Re} \int_{\Omega} \Upsilon u N(\bar{z}) d x\right|_{0} ^{T}+\operatorname{Re} \int_{0}^{T} \int_{\Omega} \Upsilon u N(\overline{\dot{z}}) d x d t \\
& =\operatorname{Re} \int_{0}^{T} \int_{\Omega} \Upsilon u N(\overline{\dot{z}}) d x d t \\
& =\operatorname{Re} \int_{0}^{T} \int_{\Omega} \operatorname{div}_{0}(\Upsilon u \overline{\dot{z}} N) d x d t-\operatorname{Re} \int_{0}^{T} \int_{\Omega} \Upsilon u \overline{\dot{z}} \operatorname{div}_{0}(N) d x d t \\
& -\operatorname{Re} \int_{0}^{T} \int_{\Omega} \overline{\dot{z}} N(\Upsilon u) d x d t \\
& =\operatorname{Re} \int_{0}^{T} \int_{\partial \Omega} \Upsilon u \overline{\dot{z}} N \cdot \nu d \Gamma d t-\operatorname{Re} \int_{0}^{T} \int_{\Omega} \Upsilon u \overline{\dot{z}} \operatorname{div}_{0}(N) d x d t \\
& -\operatorname{Re} \int_{0}^{T} \int_{\Omega} \overline{\dot{z}} N(\Upsilon u) d x d t \\
& =-\operatorname{Re} \int_{0}^{T} \int_{\Omega} \Upsilon u \overline{\dot{z}} \operatorname{div}_{0}(N) d x d t-\operatorname{Re} \int_{0}^{T} \int_{\Omega} \overline{\dot{z}} N(\Upsilon u) d x d t .
\end{aligned}
$$


By the equalities (3.20), (3.28) and the fact that $\Upsilon u \in L^{2}\left(0, T ; H^{3 / 2}(\Omega)\right)$ implies $N(\Upsilon u) \in L^{2}\left(0, T ; H^{1 / 2}(\Omega)\right)$, all continuously in $u \in L^{2}(\Sigma)$, we have

$$
-\operatorname{Re} \int_{0}^{T} \int_{\Omega} \Upsilon u_{t} N(\bar{z}) d x d t \leq C\|u\|_{L^{2}(\Sigma)}^{2} .
$$

A similar estimate holds true for the first term of $\mathrm{RHS}_{3}$ and we thereby obtain (3.23).

STEP 8. We can then extend estimate (3.23) of $\mathrm{RHS}_{3}$ and (3.24) of $b_{0, T}$ to all $u \in$ $L^{2}(\Sigma)$ by a density argument, which together with (3.19) and (3.15) gives (3.3). The proof is complete.

4. Proof of Theorem 1.2. In order to show Theorem 1.2, we need the following Lemma 4.1 which generalizes the regularity results for elliptic boundary problems to the transmission case.

LEMMA 4.1. Suppose $\phi$ solves the following equation:

$$
\left\{\begin{array}{l}
a(x) \mathcal{A}^{2} \phi(x)=h(x), x \in \Omega, \\
\phi_{2}(x)=0, \frac{\partial \phi_{2}(x)}{\partial \nu_{\mathcal{A}}}=u(x), x \in \Gamma, \\
\phi_{1}(x)=\phi_{2}(x), \frac{\partial \phi_{1}(x)}{\partial \nu_{\mathcal{A}}}=\frac{\partial \phi_{2}(x)}{\partial \nu_{\mathcal{A}}}, x \in \Gamma_{1}, \\
a_{1} \mathcal{A} \phi_{1}(x)=a_{2} \mathcal{A} \phi_{2}(x), a_{1} \frac{\partial \mathcal{A} \phi_{1}(x)}{\partial \nu_{\mathcal{A}}}=a_{2} \frac{\partial \mathcal{A} \phi_{2}(x)}{\partial \nu_{\mathcal{A}}}, x \in \Gamma_{1} .
\end{array}\right.
$$

Then there exists a constant $C>0$ independent of $(\phi, h, u)$ such that

$$
\|\phi\|_{H^{s}(\Omega)} \leq C\left(\|h\|_{H^{s-4}(\Omega)}+\|u\|_{H^{s-\frac{3}{2}(\Gamma)}}\right) \text { for } s \geq 4
$$

and

$$
\|\phi\|_{H^{s}(\Omega)} \leq C\|u\|_{H^{s-\frac{3}{2}}(\Gamma)} \text { for } 0<s<4 \text { and } h \equiv 0 .
$$

Proof. The solution of (4.1) can be written as

$$
\phi(x)= \begin{cases}\phi_{1}(x), & x \in \Omega_{1}, \\ \phi_{2}(x), & x \in \Omega_{2},\end{cases}
$$

where $\phi_{1}$ and $\phi_{2}$ satisfy, respectively,

$$
\left\{\begin{array}{l}
a_{2} \mathcal{A}^{2} \phi_{2}(x)=h(x), \quad x \in \Omega, \\
\phi_{2}(x)=0, \frac{\partial \phi_{2}(x)}{\partial \nu_{\mathcal{A}}}=u(x), \quad x \in \Gamma,
\end{array}\right.
$$

and

$$
\left\{\begin{array}{l}
a_{1} \mathcal{A}^{2} \phi_{1}(x)=h(x), x \in \Omega_{1}, \\
\phi_{1}(x)=\phi_{2}(x), \frac{\partial \phi_{1}(x)}{\partial \nu_{\mathcal{A}}}=\frac{\partial \phi_{2}(x)}{\partial \nu_{\mathcal{A}}}, \quad x \in \Gamma_{1}, \\
a_{1} \mathcal{A} \phi_{1}(x)=a_{2} \mathcal{A} \phi_{2}(x), a_{1} \frac{\partial \mathcal{A} \phi_{1}(x)}{\partial \nu_{\mathcal{A}}}=a_{2} \frac{\partial \mathcal{A} \phi_{2}(x)}{\partial \nu_{\mathcal{A}}}, \quad x \in \Gamma_{1} .
\end{array}\right.
$$


By virtue of the elliptic regularity (7.27) of [20] on p. 189, we have

$$
\left\|\phi_{2}\right\|_{H^{s}(\Omega)} \leq C\left(\|h\|_{H^{s-4}(\Omega)}+\|u\|_{H^{s-\frac{3}{2}(\Gamma)}}\right) \text { for } s \geq 4 .
$$

Similarly, by the elliptic regularity (7.28) of [20] on p. 189, we also have

$$
\left\|\phi_{2}\right\|_{H^{s}(\Omega)} \leq C\|u\|_{H^{s-\frac{3}{2}}(\Gamma)} \text { for } s<4 \text { and } h \equiv 0
$$

where $C>0$ denotes, throughout the proof, some constants independent of functions involved although it may have different values in different cases.

For $s \geq 4$, it follows from the standard trace theorem in Sobolev space that $\left.\phi_{2}\right|_{\Gamma_{1}} \in$ $H^{s-\frac{1}{2}}\left(\Gamma_{1}\right),\left.\frac{\partial \phi_{2}}{\partial \nu_{\mathcal{A}}}\right|_{\Gamma_{1}} \in H^{s-\frac{3}{2}}\left(\Gamma_{1}\right)$ and

$$
\left(\left\|\phi_{2}\right\|_{H^{s-\frac{1}{2}\left(\Gamma_{1}\right)}}+\left\|\frac{\partial \phi_{2}}{\partial \nu_{\mathcal{A}}}\right\|_{H^{s-\frac{3}{2}}\left(\Gamma_{1}\right)}\right) \leq C\left\|\phi_{2}\right\|_{H^{s}(\Omega)} \text { for } s \geq 4 .
$$

As for $0<s<4$, applying the trace theorem 7.3 of [20] on p. 187, we have $\left.\phi_{2}\right|_{\Gamma_{1}} \in$ $H^{s-\frac{1}{2}}\left(\Gamma_{1}\right),\left.\frac{\partial \phi_{2}}{\partial \nu_{\mathcal{A}}}\right|_{\Gamma_{1}} \in H^{s-\frac{3}{2}}\left(\Gamma_{1}\right)$ and

$$
\left(\left\|\phi_{2}\right\|_{H^{s-\frac{1}{2}\left(\Gamma_{1}\right)}}+\left\|\frac{\partial \phi_{2}}{\partial \nu_{\mathcal{A}}}\right\|_{H^{s-\frac{3}{2}}\left(\Gamma_{1}\right)}\right) \leq C\left\|\phi_{2}\right\|_{H^{s}\left(\Omega_{1}\right)} \text { for } 0<s<4 \text { and } h \equiv 0 .
$$

Applying the same elliptic regularity as above for both $s \geq 4$ and $0<s<4$, we get $\phi_{1} \in H^{s}\left(\Omega_{1}\right)$ for any $s>0$. At the same time, it follows from the connection boundary conditions on $\Gamma_{1}$ that

$$
\left\|\phi_{1}\right\|_{H^{s}\left(\Omega_{1}\right)} \leq C\left(\|h\|_{H^{s-4}\left(\Omega_{1}\right)}+\left\|\phi_{2}\right\|_{H^{s-\frac{1}{2}}\left(\Gamma_{1}\right)}+\left\|\frac{\partial \phi_{2}}{\partial \nu_{\mathcal{A}}}\right\|_{H^{s-\frac{3}{2}}\left(\Gamma_{1}\right)}\right) \text { for } s \geq 4
$$

and

$$
\left\|\phi_{1}\right\|_{H^{s}\left(\Omega_{1}\right)} \leq C\left(\left\|\phi_{2}\right\|_{H^{s-\frac{1}{2}}\left(\Gamma_{1}\right)}+\left\|\frac{\partial \phi_{2}}{\partial \nu_{\mathcal{A}}}\right\|_{H^{s-\frac{3}{2}}\left(\Gamma_{1}\right)}\right) \text { for } 0<s<4 \text { and } h \equiv 0 .
$$

(4.2) and (4.3) are thus obtained by combining (4.4), (4.6), (4.8), (4.5), (4.7) and (4.9).

It is known from the Appendix of [11] that the transfer function of the system (2.6) is

$$
H(\lambda)=\lambda B^{*}\left(\lambda^{2}+\tilde{A}\right)^{-1} B
$$

where $\tilde{A}, B$ and $B^{*}$ are given by $(2.3),(2.4)$ and (2.5), respectively. Moreover, from the well-posedness claimed by Theorem 1.1, it follows that there are constants $M, \beta>0$ such that $([10])$

$$
\sup _{\operatorname{Re} \lambda \geq \beta}\|H(\lambda)\|_{\mathcal{L}(U)}=M<\infty .
$$


Proposition 4.1. Theorem 1.2 is valid if for any $u \in C_{0}^{\infty}(\Gamma)$, the solution $w$ to the following equation

$$
\left\{\begin{array}{l}
\lambda^{2} w(x)+a(x) \mathcal{A}^{2} w(x)=0, x \in \Omega \\
w_{2}(x)=0, \frac{\partial w_{2}(x)}{\partial \nu_{\mathcal{A}}}=u(x), x \in \Gamma \\
w_{1}(x)=w_{2}(x), \frac{\partial w_{1}(x)}{\partial \nu_{\mathcal{A}}}=\frac{\partial w_{2}(x)}{\partial \nu_{\mathcal{A}}}, x \in \Gamma_{1} \\
a_{1} \mathcal{A} w_{1}(x)=a_{2} \mathcal{A} w_{2}(x), a_{1} \frac{\partial \mathcal{A} w_{1}(x)}{\partial \nu_{\mathcal{A}}}=a_{2} \frac{\partial \mathcal{A} w_{2}(x)}{\partial \nu_{\mathcal{A}}}, x \in \Gamma_{1}
\end{array}\right.
$$

satisfies

$$
\lim _{\lambda \in \mathbb{R}, \lambda \rightarrow+\infty} \int_{\Gamma} a_{2}\left|\frac{1}{\lambda} \mathcal{A} w(x)\right|^{2} d x=0 .
$$

Proof. It was shown in [29] that in the frequency domain, (1.7) is equivalent to

$$
\lim _{\lambda \in \mathbb{R}, \lambda \rightarrow+\infty} H(\lambda) u=0 \text { in strong topology of } U \text { for any } u \in U,
$$

where $H(\lambda)$ is given by (4.10). Due to (4.11) and the density argument, it suffices to show that (4.14) is satisfied for all $u \in C_{0}^{\infty}(\Gamma)$.

Now assume that $u \in C_{0}^{\infty}(\Gamma)$, and put

$$
w(x)=\left(\left(\lambda^{2}+\tilde{A}\right)^{-1} B u\right)(x) .
$$

Then $w$ satisfies (4.12) and

$$
(H(\lambda) u)(x)=-\lambda \mathcal{A}\left(\mathscr{A}^{-1} w\right)(x), x \in \Gamma .
$$

Taking $s=4$ in (4.2), we get a function $v \in H^{4}\left(\Omega, \Gamma_{1}\right)$ satisfying the following equation:

$$
\left\{\begin{array}{l}
a(x) \mathcal{A}^{2} v(x)=0, x \in \Omega, \\
v_{2}(x)=0, \frac{\partial v_{2}(x)}{\partial \nu_{\mathcal{A}}}=u(x), \quad x \in \Gamma, \\
v_{1}(x)=v_{2}(x), \frac{\partial v_{1}(x)}{\partial \nu_{\mathcal{A}}}=\frac{\partial v_{2}(x)}{\partial \nu_{\mathcal{A}}}, \quad x \in \Gamma_{1}, \\
a_{1} \mathcal{A} v_{1}(x)=a_{2} \mathcal{A} v_{2}(x), a_{1} \frac{\partial \mathcal{A} v_{1}(x)}{\partial \nu_{\mathcal{A}}}=a_{2} \frac{\partial \mathcal{A} v_{2}(x)}{\partial \nu_{\mathcal{A}}}, x \in \Gamma_{1} .
\end{array}\right.
$$

Thus (4.12) can be written as

$$
\left\{\begin{array}{l}
\lambda^{2} w(x)+a(x) \mathcal{A}^{2}(w(x)-v(x))=0, x \in \Omega, \\
\left(w_{2}-v_{2}\right)(x)=\frac{\partial\left(w_{2}-v_{2}\right)(x)}{\partial \nu_{\mathcal{A}}}=0, x \in \Gamma, \\
\left(w_{1}-v_{1}\right)(x)=\left(w_{2}-v_{2}\right)(x), \frac{\partial\left(w_{1}-v_{1}\right)(x)}{\partial \nu_{\mathcal{A}}}=\frac{\partial\left(w_{2}-v_{2}\right)(x)}{\partial \nu_{\mathcal{A}}}, x \in \Gamma_{1}, \\
a_{1} \mathcal{A}\left(w_{1}-v_{1}\right)(x)=a_{2} \mathcal{A}\left(w_{2}-v_{2}\right)(x), \\
a_{1} \frac{\partial \mathcal{A}\left(w_{1}-v_{1}\right)(x)}{\partial \nu_{\mathcal{A}}}=a_{2} \frac{\partial \mathcal{A}\left(w_{2}-v_{2}\right)(x)}{\partial \nu_{\mathcal{A}}}, x \in \Gamma_{1}
\end{array}\right.
$$


or

$$
\lambda^{2}\left(\mathscr{A}^{-1} w\right)(x)=a(x)(-w(x)+v(x)) .
$$

So (4.15) becomes

$$
(H(\lambda) u)(x)=\frac{1}{\lambda} a(x) \mathcal{A} w(x)-\frac{1}{\lambda} a(x) \mathcal{A} v(x)
$$

Since $\mathcal{A} v(x)$ is independent of $\lambda$, the required result then follows from (4.16) and (4.14).

In order to prove (4.13), we state the following Lemma 4.2 that comes from Lemma 4.1 of $[16]$.

LEmma 4.2. Let $w$ be the solution of (4.12). Then there exists a function $\eta(x)$ independent of $\lambda$, which is continuous on $\Gamma$, such that

$$
\Delta_{g} w(x)=\frac{\partial^{2} w(x)}{\partial \mu^{2}}+\eta(x) \frac{\partial w(x)}{\partial \mu}, \forall x \in \Gamma .
$$

Now we are in a position to prove Theorem 1.2.

Proof. Firstly, multiply both sides of the first equation of (4.12) by $\bar{w}$ and integrate by parts to give

$$
\begin{aligned}
0 & =\int_{\Omega} \lambda^{2}|w|^{2}+a(x) \mathcal{A}^{2} w \cdot \bar{w} d x \\
& =\int_{\Omega} \lambda^{2}|w|^{2} d x+\int_{\Omega} a(x)|\mathcal{A} w|^{2} d x-\int_{\Gamma} a_{2} \mathcal{A} w_{2} \frac{\partial \bar{w}_{2}}{\partial \nu_{\mathcal{A}}} d \Gamma \\
& =\int_{\Omega} \lambda^{2}|w|^{2} d x+\int_{\Omega} a(x)|\mathcal{A} w|^{2} d x-\int_{\Gamma} a_{2} \mathcal{A} w_{2} \bar{u} d \Gamma
\end{aligned}
$$

from which we get

$$
\int_{\Omega}|w|^{2} d x+\frac{1}{\lambda^{2}} \int_{\Omega} a(x)|\mathcal{A} w|^{2} d x \leq \frac{1}{\lambda}\|u\|_{L^{2}(\Gamma)}\left\|\frac{1}{\lambda} a_{2} \mathcal{A} w_{2}\right\|_{L^{2}(\Gamma)} .
$$

Secondly, choose the vector field $N$ on $\bar{\Omega}$ as in (3.4). As was done in Section 3, multiply both sides of the first equation of (4.12) by $N(\bar{w})$, integrate by parts and use (2.8), (2.9), 
(3.7), (3.10), (3.17), (4.17) and the divergence formula to yield

$$
\begin{aligned}
0= & \operatorname{Re} \int_{\Omega}\left[\lambda^{2} w N(\bar{w})+a(x) \mathcal{A}^{2} w N(\bar{w})\right] d x \\
= & \frac{\lambda^{2}}{2} \int_{\Omega}\left[\operatorname{div}_{0}\left(|w|^{2} N\right)-|w|^{2} \operatorname{div}_{0}(N)\right] d x+\operatorname{Re} \int_{\Omega} a(x) \mathcal{A} w \Delta_{g}(N(\bar{w})) d x \\
& +\operatorname{Re} \int_{\Gamma} N\left(\bar{w}_{2}\right) a_{2} \frac{\partial\left(\mathcal{A} w_{2}\right)}{\partial \mu} d \Gamma+\operatorname{Re} \int_{\Gamma_{1}} N\left(\bar{w}_{2}\right) a_{2} \frac{\partial\left(\mathcal{A} w_{2}\right)}{\partial \mu} d \Gamma \\
& -\operatorname{Re} \int_{\Gamma_{1}} N\left(\bar{w}_{1}\right) a_{1} \frac{\partial\left(\mathcal{A} w_{1}\right)}{\partial \mu} d \Gamma-\operatorname{Re} \int_{\Gamma} a_{2} \mathcal{A} w_{2} \frac{\partial\left(N\left(\bar{w}_{2}\right)\right)}{\partial \mu} d \Gamma \\
& -\operatorname{Re} \int_{\Gamma_{1}} a_{2} \mathcal{A} w_{2} \frac{\partial\left(N\left(\bar{w}_{2}\right)\right)}{\partial \mu} d \Gamma+\operatorname{Re} \int_{\Gamma_{1}} a_{1} \mathcal{A} w_{1} \frac{\partial\left(N\left(\bar{w}_{1}\right)\right)}{\partial \mu} d \Gamma \\
& +\operatorname{Re} \int_{\Omega} a(x) D f(\mathcal{A} w) N(\bar{w}) d x \\
= & -\frac{\lambda^{2}}{2} \int_{\Omega} \operatorname{div}_{0}(N)|w|^{2} d x+\operatorname{Re} \int_{\Omega} a(x) \mathcal{A} w \Delta_{g}(N(\bar{w})) d x \\
& +\operatorname{Re} \int_{\Gamma} \frac{\bar{u}}{\left|\nu_{\mathcal{A}}\right|_{g}} a_{2} \frac{\partial\left(\mathcal{A} w_{2}\right)}{\partial \mu} d \Gamma-\operatorname{Re} \int_{\Gamma} a_{2} \mathcal{A} w_{2} \frac{\partial^{2} \bar{w}_{2}}{\partial \mu^{2}} d \Gamma \\
& +\operatorname{Re} \int_{\Gamma_{1}}\left[a_{1}\left|\mathcal{A} w_{1}\right|^{2}-a_{2}\left|\mathcal{A} w_{2}\right|^{2}\right] d \Gamma+\operatorname{Re} \int_{\Gamma} N\left(\bar{w}_{2}\right) a_{2} \mathcal{A} w_{2} D f \cdot \nu d \Gamma \\
& -\operatorname{Re} \int_{\Omega} a(x) \mathcal{A} w D f(N(\bar{w})) d x-\operatorname{Re} \int_{\Omega} a(x) N(\bar{w}) \mathcal{A} w \operatorname{div} v_{0}(D f) d x .
\end{aligned}
$$

Hence

$$
\begin{aligned}
a_{2} & \left\|\frac{1}{\lambda} \mathcal{A} w\right\|_{L^{2}(\Gamma)}^{2}=-\int_{\Omega} \operatorname{div}_{0}(N)|w|^{2} d x+\frac{2}{\lambda^{2}} \operatorname{Re} \int_{\Gamma} \frac{\bar{u}}{\left|\nu_{\mathcal{A}}\right|_{g}} a_{2} \frac{\partial\left(\mathcal{A} w_{2}\right)}{\partial \mu} d \Gamma \\
& +\frac{2}{\lambda^{2}} \operatorname{Re} \int_{\Gamma} \frac{\bar{u}}{\left|\nu_{\mathcal{A}}\right|_{g}} \eta(x) a_{2} \mathcal{A} w_{2} d \Gamma+\frac{2}{\lambda^{2}} \operatorname{Re} \int_{\Gamma} \frac{\bar{u}}{\left|\nu_{\mathcal{A}}\right|_{g}} a_{2} \mathcal{A} w_{2} D f \cdot \nu d \Gamma \\
& +\frac{2}{\lambda^{2}} \operatorname{Re} \int_{\Gamma} a_{2} \mathcal{A} w_{2} D f\left(\bar{w}_{2}\right) d \Gamma-\frac{1}{\lambda^{2}} \int_{\Omega} a(x)|\mathcal{A} w|^{2} \operatorname{div}_{g}(N) d x \\
& +\frac{2}{\lambda^{2}} \operatorname{Re} \int_{\Omega} a(x) \mathcal{A} w\left[(-\triangle N)(\bar{w})+2\left\langle D N, D^{2} \bar{w}\right\rangle_{T^{2}\left(\mathbb{R}_{x}^{2}\right)}-D^{2} f(N, D \bar{w})\right. \\
& \left.-D^{2} \bar{w}(N, D f)+\operatorname{Ric}(N, D \bar{w})\right] d x-\frac{2}{\lambda^{2}} \operatorname{Re} \int_{\Omega} a(x) \mathcal{A} w D f(N(\bar{w})) d x \\
& -\frac{2}{\lambda^{2}} \operatorname{Re} \int_{\Omega} a(x) N(\bar{w}) \mathcal{A} w \operatorname{div}_{0}(D f) d x+\frac{1}{2} \int_{\Gamma_{1}} \frac{\left(a_{2}-a_{1}\right)}{a_{1} a_{2}}\left|a_{1} \mathcal{A} w_{1}\right|^{2} d \Gamma \\
\leq & C_{1}\|w\|_{L^{2}(\Omega)}^{2}+\frac{C_{2}}{\lambda^{2}}\|u\|_{L^{2}(\Gamma)}\|w\|_{H^{4}(\Omega)}+\frac{C_{3}}{\lambda}\|u\|_{L^{2}(\Gamma)}\left\|\frac{1}{\lambda} \mathcal{A} w\right\|_{L^{2}(\Omega)}^{2} \\
& +\frac{C_{4}}{\lambda^{2}}\|\mathcal{A} w\|_{L^{2}(\Omega)}^{2},
\end{aligned}
$$


where $C_{i}>0, i=1,2,3,4$ are constants independent of $\lambda$. Notice that in the last inequality above, we have used Lemma 2.2, the assumption $a_{2} \leq a_{1}$ and the following facts:

$$
\begin{aligned}
& \sup _{x \in \Gamma}|\eta(x)| \leq C,\|D f(w)\|_{L^{2}(\Gamma)} \leq C\|u\|_{L^{2}(\Gamma)}, \\
& \|w\|_{H^{2}(\Omega)} \leq C\|\mathcal{A} w\|_{L^{2}(\Omega)},\left\|\frac{\partial(\mathcal{A} w)}{\partial \mu}\right\|_{L^{2}(\Gamma)} \leq C\|w\|_{H^{4}(\Omega)},
\end{aligned}
$$

for some constant $C>0$ independent of $\lambda$. The first two inequalities are apparent. The third estimate is also well known due to the vanishing condition of $w$ on $\partial \Omega$, and the last estimate comes from the trace theorem in Sobolev space (see [20]).

Finally, setting $s=4$ in (4.2) we deduce that the solution of (4.12) satisfies

$$
\|w\|_{H^{4}(\Omega)} \leq C_{5}\left[\left\|\frac{1}{\lambda^{2}} w\right\|_{L^{2}(\Omega)}+\|u\|_{H^{5 / 2}(\Gamma)}\right]
$$

for some constant $C_{5}$ independent of $\lambda$. This together with (4.18) and (4.19) yields

$$
\begin{aligned}
a_{2}\left\|\frac{1}{\lambda} \mathcal{A} w\right\|_{L^{2}(\Gamma)}^{2} \leq & \left(C_{1}+C_{3}+C_{4}\right) \frac{1}{\lambda}\|u\|_{L^{2}(\Gamma)}\left\|\frac{1}{\lambda} \mathcal{A} w\right\|_{L^{2}(\Gamma)}+C_{2} C_{5}\|u\|_{L^{2}(\Gamma)}\|w\|_{L^{2}(\Omega)} \\
& +C_{2} C_{5} \frac{1}{\lambda^{2}}\|u\|_{L^{2}(\Gamma)}\|u\|_{H^{5 / 2}(\Gamma)} \\
\leq & \left(C_{1}+C_{3}+C_{4}\right) \frac{1}{\lambda}\|u\|_{L^{2}(\Gamma)}\left\|\frac{1}{\lambda} \mathcal{A} w\right\|_{L^{2}(\Gamma)} \\
& +C_{2} C_{5} \lambda^{-1 / 2}\|u\|_{L^{2}(\Gamma)}^{3 / 2}\left\|\frac{1}{\lambda} \mathcal{A} w\right\|_{L^{2}(\Omega)}^{1 / 2}+C_{2} C_{5} \frac{1}{\lambda^{2}}\|u\|_{L^{2}(\Gamma)}\|u\|_{H^{5 / 2}(\Gamma)},
\end{aligned}
$$

which implies that $\varlimsup_{\lambda \in \mathbb{R}, \lambda \rightarrow+\infty} a_{2}\left\|\lambda^{-1} \mathcal{A} w\right\|_{L^{2}(\Gamma)}<+\infty$. Therefore

$$
\lim _{\lambda \in \mathbb{R}, \lambda \rightarrow+\infty} a_{2}\left\|\frac{1}{\lambda} \mathcal{A} w\right\|_{L^{2}(\Gamma)}=0 .
$$

This is (4.13). The proof is complete.

5. Exact controllability. For the controllability problem, we confine the control to be real functions. We first consider the well-posedness and boundary regularity of the dual system of problem (1.1):

$$
\left\{\begin{array}{l}
\ddot{w}(x, t)+a(x) \mathcal{A}^{2} w(x, t)=0, \quad(x, t) \in Q, \\
w(x, 0)=w^{0}, \dot{w}(x, 0)=w^{1}, \quad x \in \Omega \\
w_{2}(x, t)=0, \frac{\partial w_{2}}{\partial \nu_{\mathcal{A}}}(x, t)=0, \quad(x, t) \in \Sigma \\
w_{1}(x, t)=w_{2}(x, t), \frac{\partial w_{1}}{\partial \nu_{\mathcal{A}}}(x, t)=\frac{\partial w_{2}}{\partial \nu_{\mathcal{A}}}(x, t), \quad(x, t) \in \Sigma_{1}, \\
a_{1} \mathcal{A} w_{1}(x, t)=a_{2} \mathcal{A} w_{2}(x, t), a_{1} \frac{\partial \mathcal{A} w_{1}}{\partial \nu_{\mathcal{A}}}(x, t)=a_{2} \frac{\partial \mathcal{A} w_{2}}{\partial \nu_{\mathcal{A}}}(x, t), \quad(x, t) \in \Sigma_{1} .
\end{array}\right.
$$


Define operator $\mathbb{A}$ by

$$
\mathbb{A}=\left(\begin{array}{cc}
0 & I \\
-a \mathscr{A} & 0
\end{array}\right) \text { with } D(\mathbb{A})=H^{4}\left(\Omega, \Gamma_{1}\right) \times H_{0}^{2}(\Omega) .
$$

It is readily shown that $\mathbb{A}$ is skew-adjoint, i.e. $\mathbb{A}^{*}=-\mathbb{A}$. So $\mathbb{A}$ generates a $C_{0}$-group $e^{\mathbb{A} t}$ on $H_{0}^{2}(\Omega) \times L^{2}(\Omega)$.

Proposition 5.1. For any given initial data $\left(w^{0}, w^{1}\right) \in H_{0}^{2}(\Omega) \times L^{2}(\Omega)$, Equation (5.1) admits a unique weak solution

$$
w(t) \in C\left([0, T] ; H_{0}^{2}(\Omega)\right) \cap C^{1}\left([0, T] ; L^{2}(\Omega)\right) .
$$

Furthermore, if $\left(w^{0}, w^{1}\right) \in H^{4}\left(\Omega, \Gamma_{1}\right) \times H_{0}^{2}(\Omega)$, then

$$
w(t) \in C\left([0, T] ; H^{4}\left(\Omega, \Gamma_{1}\right)\right) \cap C^{1}\left([0, T] ; H_{0}^{2}(\Omega)\right) .
$$

To discuss the boundary regularity of the solution of (5.1), we need the following Lemma 5.1 that is similar to Lemma 4.2 .

LEMma 5.1. Let $w$ be a smooth function on $\bar{\Omega}$ such that $\left.w\right|_{\Gamma}=0$. Then there exists a continuous function $m(x)$ on $\Gamma$ independent of $w$ such that

$$
\Delta_{g} w(x)=\frac{\partial^{2} w(x)}{\partial \mu^{2}}+m(x) \frac{\partial w(w)}{\partial \mu}, \forall x \in \Gamma .
$$

Furthermore, if $w$ satisfies $\left.\frac{\partial w}{\partial \nu_{\mathcal{A}}}\right|_{\Gamma}=0$, then

$$
\left.N(w)\right|_{\Gamma}=0 \text { for any vector field } N \text { on } \bar{\Omega} \text {. }
$$

By Lemma 5.1,

$$
\mathcal{A} w=\Delta_{g} w+D f(w)=\Delta_{g} w=\frac{\partial^{2} w}{\partial \mu^{2}}=\frac{1}{\left|\nu_{\mathcal{A}}\right|_{g}^{2}} \frac{\partial^{2} w}{\partial \nu_{\mathcal{A}}^{2}} \text { on } \Gamma .
$$

Now, we establish the following multiplier identity that plays a key role in obtaining the boundary regularity and the observability inequality.

Lemma 5.2. Let $N$ be a vector field on $\left(\mathbb{R}^{n}, g\right)$. Assume that $w$ solves problem (5.1). Let $f(x)=\frac{1}{2} \log \operatorname{det}\left(a_{i j}(x)\right)$. Then

$$
\begin{aligned}
& \frac{1}{2} \int_{\Sigma} a_{2}\left(\mathcal{A} w_{2}\right)^{2} N \cdot \nu d \Sigma-\frac{1}{2} \int_{\Sigma_{1}}\left[a_{1}\left(\mathcal{A} w_{1}\right)^{2}-a_{2}\left(\mathcal{A} w_{2}\right)^{2}\right] N \cdot \nu d \Sigma \\
& =\left.\int_{\Omega} \dot{w} N(w) d x\right|_{0} ^{T}+\frac{1}{2} \int_{Q}\left[|\dot{w}|^{2}-a(x)(\mathcal{A} w)^{2}\right] \operatorname{div}_{0} N d Q \\
& +\int_{Q} a(x) \mathcal{A} w\left[2\left\langle D N, D^{2} w\right\rangle_{T^{2}\left(\mathbb{R}_{x}^{n}\right)}+\mathcal{F} N(w)+\operatorname{Ric}(N, D w)-D^{2} f(N, D w)\right] d Q
\end{aligned}
$$

where $\mathcal{F} N \in \mathfrak{X}\left(\mathbb{R}^{n}\right)$ is defined by $\mathcal{F} N=D_{D f} N-\triangle N$ and $D^{2} f$ is the Hessian of $f$ in terms of $g$. 
Proof. Multiply the first equation of (5.1) by $N(w)$ and integrate by parts to obtain

$$
\begin{aligned}
\int_{Q} \ddot{w} N(w) d Q=\left.\int_{\Omega} \dot{w} N(w) d x\right|_{0} ^{T}-\int_{Q} \dot{w} N(\dot{w}) d Q \\
=\left.\int_{\Omega} \dot{w} N(w) d x\right|_{0} ^{T}-\int_{Q_{1}} \dot{w}_{1} N\left(\dot{w}_{1}\right) d Q-\int_{Q_{2}} \dot{w}_{2} N\left(\dot{w}_{2}\right) d Q \\
=\left.\int_{\Omega} \dot{w} N(w) d x\right|_{0} ^{T}-\frac{1}{2} \int_{Q_{1}}\left[\operatorname{div}_{0}\left(\left|\dot{w}_{1}\right|^{2} N\right)-\left|\dot{w}_{1}\right|^{2} \operatorname{div}_{0} N\right] d Q \\
-\frac{1}{2} \int_{Q_{2}}\left[\operatorname{div}_{0}\left(\left|\dot{w}_{2}\right|^{2} N\right)-\left|\dot{w}_{2}\right|^{2} \operatorname{div}_{0} N\right] d Q \\
=\left.\int_{\Omega} \dot{w} N(w) d x\right|_{0} ^{T}-\frac{1}{2} \int_{\Sigma_{1}}\left|\dot{w}_{1}\right|^{2} N \cdot(-\nu) d \Sigma+\frac{1}{2} \int_{Q_{1}}\left|\dot{w}_{1}\right|^{2} \operatorname{div}_{0} N d Q \\
-\frac{1}{2} \int_{\Sigma_{1}}\left|\dot{w}_{2}\right|^{2} N \cdot \nu d \Sigma-\frac{1}{2} \int_{\Sigma}\left|\dot{w}_{2}\right|^{2} N \cdot \nu d \Sigma+\frac{1}{2} \int_{Q_{2}}\left|\dot{w}_{2}\right|^{2} \operatorname{div}_{0} N d Q \\
=\left.\int_{\Omega} \dot{w} N(w) d x\right|_{0} ^{T}+\frac{1}{2} \int_{Q}|\dot{w}|^{2} \operatorname{div}_{0} N d Q .
\end{aligned}
$$

Given $x \in \mathbb{R}^{n}$. Let $E_{1}, E_{2}, \cdots, E_{n}$ be a frame field normal at $x$ on $\left(\mathbb{R}^{n}, g\right)$, which means that $\left\langle E_{i}, E_{j}\right\rangle_{g}=\delta_{i j}$ in some neighborhood of $x$ and $\left(D_{E_{i}} E_{j}\right)(x)=0$ for $1 \leq i, j \leq n$ (see [33]). Let $N=\sum_{i=1}^{n} \gamma_{i} E_{i}$. Then $N(w)=\sum_{i=1}^{n} \gamma_{i} E_{i}(w)$, where $E_{i}(w)$ is the covariant derivative of $w$ with respect to $E_{i}$ under the Riemannian metric $g$. Then at $x$

$$
\begin{aligned}
N(\mathcal{A} w) & =N\left(\Delta_{g} w+(D f) w\right)=N\left(\Delta_{g} w\right)+N\left(\langle D f, D w\rangle_{g}\right) \\
& =N\left(\Delta_{g} w\right)+N\left(\left\langle E_{i}(f) E_{i}, E_{j}(w) E_{j}\right\rangle_{g}\right) \\
& =N\left(\Delta_{g} w\right)+\gamma_{j} E_{j}\left(E_{i}(f) E_{i}(w)\right) \\
& =N\left(\Delta_{g} w\right)+\gamma_{j} E_{j} E_{i}(f) E_{i}(w)+\gamma_{j} E_{i}(f) E_{j} E_{i}(w) \\
& =N\left(\Delta_{g} w\right)+D^{2} f(N, D w)+D^{2} w(N, D f),
\end{aligned}
$$

where $E_{j} E_{i}(f)$ and $E_{j} E_{i}(w)$ are, respectively, the second covariant derivatives of $f$ and $w$ at $x$, and

$$
\begin{aligned}
\langle D f, D(N(w))\rangle_{g} & =E_{i}(f) E_{i}(N(w))=E_{i}(f)\left[E_{i}\left(\gamma_{j}\right) E_{j}(w)+\gamma_{j} E_{i} E_{j}(w)\right] \\
& =D N(D w, D f)+D^{2} w(N, D f)
\end{aligned}
$$

With the help of (2.8), (5.10) and (5.11), we obtain

$$
\begin{aligned}
& \mathcal{A}(N(w))=\left(\Delta_{g}+D f\right)(N(w))=\Delta_{g}(N(w))+\langle D(N(w)), D f\rangle_{g} \\
&=(-\triangle N)(w)+2\left\langle D N, D^{2} w\right\rangle_{T^{2}\left(\mathbb{R}_{x}^{n}\right)}+N\left(\Delta_{g} w\right)+\operatorname{Ric}(N, D w) \\
&+D N(D w, D f)+D^{2} w(N, D f) \\
&= N(\mathcal{A} w)+2\left\langle D N, D^{2} w\right\rangle_{T^{2}\left(\mathbb{R}_{x}^{n}\right)}+\mathcal{F} N(w)+\operatorname{Ric}(N, D w)-D^{2} f(N, D w),
\end{aligned}
$$


where we denoted $\mathcal{F} N=D_{D f} N-\triangle N$. By virtue of the divergence theorem,

$$
\begin{aligned}
& \int_{\Omega} a(x) N\left((\mathcal{A} w)^{2}\right) d x \\
= & \int_{\Gamma} a_{2}\left(\mathcal{A} w_{2}\right)^{2} N \cdot \nu d \Gamma+\int_{\Gamma_{1}}\left[a_{2}\left(\mathcal{A} w_{2}\right)^{2}-a_{1}\left(\mathcal{A} w_{1}\right)^{2}\right] N \cdot \nu d \Gamma \\
& -\int_{\Omega} a(x)(\mathcal{A} w)^{2} \operatorname{div}_{0} N d x .
\end{aligned}
$$

Since $w \in H_{0}^{2}(\Omega)$, by Green's identity, it follows from (5.12) and (5.13) that

$$
\begin{array}{rl}
\int_{Q} & a(x) \mathcal{A}^{2} w N(w) d Q=\int_{Q_{1}} a_{1} \mathcal{A}^{2} w_{1} N\left(w_{1}\right) d Q+\int_{Q_{2}} a_{2} \mathcal{A}^{2} w_{2} N\left(w_{2}\right) d Q \\
= & -\int_{\Sigma_{1}} N\left(w_{1}\right) a_{1} \frac{\partial \mathcal{A} w_{1}}{\partial \nu_{\mathcal{A}}} d \Sigma-\int_{Q_{1}} a_{1}\left\langle D\left(\mathcal{A} w_{1}\right), D\left(N\left(w_{1}\right)\right)\right\rangle_{g} d Q \\
& +\int_{\Sigma_{1}} N\left(w_{2}\right) a_{2} \frac{\partial \mathcal{A} w_{2}}{\partial \nu_{\mathcal{A}}} d \Sigma+\int_{\Sigma} N\left(w_{2}\right) a_{2} \frac{\partial \mathcal{A} w_{2}}{\partial \nu_{\mathcal{A}}} d \Sigma \\
& -\int_{Q_{2}} a_{2}\left\langle D\left(\mathcal{A} w_{2}\right), D\left(N\left(w_{2}\right)\right)\right\rangle_{g} d Q \\
= & \int_{\Sigma} N\left(w_{2}\right) a_{2} \frac{\partial \mathcal{A} w_{2}}{\partial \nu_{\mathcal{A}}} d \Sigma+\int_{\Sigma_{1}}\left[{ }\left(w_{2}\right) a_{2} \frac{\partial \mathcal{A} w_{2}}{\partial \nu_{\mathcal{A}}}-N\left(w_{1}\right) a_{1} \frac{\partial \mathcal{A} w_{1}}{\partial \nu_{\mathcal{A}}}\right] d \Sigma \\
& +\int_{Q_{1}} a_{1} \mathcal{A} w \mathcal{A}\left(N\left(w_{1}\right)\right) d Q+\int_{\Sigma_{1}} a_{1} \mathcal{A} w_{1} \frac{\partial N\left(w_{1}\right)}{\partial \nu_{\mathcal{A}}} d \Sigma \\
& +\int_{Q_{2}} a_{2} \mathcal{A} w_{2} \mathcal{A}\left(N\left(w_{2}\right)\right) d Q-\int_{\Sigma_{1}} a_{2} \mathcal{A} w_{2} \frac{\partial N\left(w_{2}\right)}{\partial \nu_{\mathcal{A}}} d \Sigma-\int_{\Sigma} a_{2} \mathcal{A} w_{2} \frac{\partial N\left(w_{2}\right)}{\partial \nu_{\mathcal{A}}} d \Sigma \\
= & -\int_{\Sigma} a_{2} \mathcal{A} w_{2} \frac{\partial N\left(w_{2}\right)}{\partial \nu_{\mathcal{A}}} d \Sigma+\int_{\Sigma_{1}}\left[a_{1} \mathcal{A} w_{1} \frac{\partial N\left(w_{1}\right)}{\partial \nu_{\mathcal{A}}}-a_{2} \mathcal{A} w_{2} \frac{\partial N\left(w_{2}\right)}{\partial \nu_{\mathcal{A}}}\right] d \Sigma \\
& +\int_{Q} a(x) \mathcal{A} w\left[2\left\langle D N, D^{2} w\right\rangle_{T^{2}\left(\mathbb{R}_{x}^{n}\right)}+\mathcal{A} N(w)+\operatorname{Ric}(N, D w)\right. \\
& \left.-D^{2} f(N, D w)\right] d Q+\frac{1}{2} \int_{\Sigma_{1}}\left[a_{2}\left(\mathcal{A} w_{2}\right)^{2}-a_{1}\left(\mathcal{A} w_{1}\right)^{2}\right] N \cdot \nu d \Sigma \\
& +\frac{1}{2} \int_{\Sigma} a_{2}\left(\mathcal{A} w_{2}\right)^{2} N \cdot \nu d \Sigma-\frac{1}{2} \int_{Q}(x)(\mathcal{A} w)^{2} \operatorname{div} v_{0} N d Q .
\end{array}
$$

Notice that in the last step, we used the fact that $N\left(w_{1}\right)=N\left(w_{2}\right)$ on $\Gamma_{1}$.

Since $\left.w_{2}\right|_{\Sigma}=\left.\frac{\partial w_{2}}{\partial \nu_{\mathcal{A}}}\right|_{\Sigma}=0$ implies $\left.N\left(w_{2}\right)\right|_{\Sigma}=0$, it follows from identity (5.7) that

$$
\begin{aligned}
& \frac{\partial N\left(w_{2}\right)}{\partial \nu_{\mathcal{A}}}=N\left(\frac{\partial w_{2}}{\partial \nu_{\mathcal{A}}}\right)=\left\langle N, \frac{\nu_{\mathcal{A}}}{\left|\nu_{\mathcal{A}}\right|_{g}}\right\rangle_{g} \frac{\nu_{\mathcal{A}}}{\left|\nu_{\mathcal{A}}\right|_{g}}\left(\frac{\partial w_{2}}{\partial \nu_{\mathcal{A}}}\right) \\
& =N \cdot \nu \frac{1}{\left|\nu_{\mathcal{A}}\right|_{g}^{2}} \frac{\partial^{2} w_{2}}{\partial \nu_{\mathcal{A}}^{2}}=\mathcal{A} w_{2} N \cdot \nu \text { on } \Sigma .
\end{aligned}
$$


Now we treat the term $a_{1} \mathcal{A} w_{1} \frac{\partial N\left(w_{1}\right)}{\partial \nu_{\mathcal{A}}}-a_{2} \mathcal{A} w_{2} \frac{\partial N\left(w_{2}\right)}{\partial \nu_{\mathcal{A}}}$ on $\Sigma_{1}$. Since $\left.a_{1} \mathcal{A} w_{1}\right|_{\Sigma_{1}}=$ $\left.a_{2} \mathcal{A} w_{2}\right|_{\Sigma_{1}},\left.\left(w_{1}-w_{2}\right)\right|_{\Sigma_{1}}=\left.\frac{\partial\left(w_{1}-w_{2}\right)}{\partial \nu_{\mathcal{A}}}\right|_{\Sigma_{1}}=0$, similar to $(5.15)$, we have

$$
a_{1} \mathcal{A} w_{1} \frac{\partial N\left(w_{1}\right)}{\partial \nu_{\mathcal{A}}}-a_{2} \mathcal{A} w_{2} \frac{\partial N\left(w_{2}\right)}{\partial \nu_{\mathcal{A}}}=\left[a_{1}\left(\mathcal{A} w_{1}\right)^{2}-a_{2}\left(\mathcal{A} w_{2}\right)^{2}\right] N \cdot \nu \text { on } \Sigma_{1} .
$$

Combining (5.9), (5.14), (5.15) and (5.16), we arrive at (5.8). The proof is complete.

The following Lemma 5.3 claims the boundary regularity (hidden regularity) for problem (5.1).

LEmma 5.3. There exists a constant $C_{T}>0$ such that for any mild solution of (5.1), it has

$$
\int_{\Sigma}\left(\mathcal{A} w_{2}\right)^{2} d \Sigma \leq C_{T}\left(\left\|w^{0}\right\|_{H_{0}^{2}(\Omega)}^{2}+\left\|w^{1}\right\|_{L^{2}(\Omega)}^{2}\right) .
$$

Proof. Choose the vector field $N$ in (5.8) such that $N=\nu_{\mathcal{A}}$ on $\Gamma$ and $N=0$ in some neighborhood of $\bar{\Omega}_{1}$. Then $N \cdot \nu=\left|\nu_{\mathcal{A}}\right|_{g}^{2} \geq \lambda>0$. This together with (5.8), Lemma 5.2 and Lemma 5.3 gives (5.17).

Lemma 5.4. Let $N$ be a vector field and $w$ a solution to (5.1). If $\varphi \in C^{2}\left(\mathbb{R}^{n}\right)$, then it has

$$
\int_{Q}\left[|\dot{w}|^{2}-a(x)(\mathcal{A} w)^{2}\right] \varphi d Q=-\left.\int_{\Omega} \dot{w} w \varphi d x\right|_{0} ^{T}+\int_{Q} a(x) \mathcal{A} w[w \mathcal{A} \varphi+2 D \varphi(w)] d Q .
$$

Proof. By virtue of Green's identity, we have

$$
\begin{aligned}
\left.\int_{\Omega} \dot{w} w \varphi d x\right|_{0} ^{T}=\int_{Q}\left[\left(-a(x) \mathcal{A}^{2} w\right) w \varphi+|\dot{w}|^{2} \varphi\right] d Q \\
=-\int_{Q} a(x) \mathcal{A} w \mathcal{A}(w \varphi) d Q-\int_{\Sigma} a_{2} \frac{\partial \mathcal{A} w_{2}}{\partial \nu_{\mathcal{A}}} w_{2} \varphi d \Sigma+\int_{\Sigma} a_{2} \mathcal{A} w_{2} \frac{\partial\left(w_{2} \varphi\right)}{\partial \nu_{\mathcal{A}}} d \Sigma \\
\quad+\int_{Q}|\dot{w}|^{2} \varphi d Q \\
=\int_{Q}\left[|\dot{w}|^{2}-a(x)(\mathcal{A} w)^{2}\right] \varphi d Q-\int_{Q} a(x) \mathcal{A} w[w \mathcal{A} \varphi+2 D \varphi(w)] d Q \\
\quad-\int_{\Sigma} a_{2} \frac{\partial \mathcal{A} w_{2}}{\partial \nu_{\mathcal{A}}} w_{2} \varphi d \Sigma+\int_{\Sigma} a_{2} \mathcal{A} w_{2} \frac{\partial\left(w_{2} \varphi\right)}{\partial \nu_{\mathcal{A}}} d \Sigma \\
=\int_{Q}\left[|\dot{w}|^{2}-a(x)(\mathcal{A} w)^{2}\right] \varphi d Q-\int_{Q} a(x) \mathcal{A} w[w \mathcal{A} \varphi+2 D \varphi(w)] d Q
\end{aligned}
$$

In the last step above, we used the following formula:

$$
\mathcal{A}(w \varphi)=(\mathcal{A} w) \varphi+2 D \varphi(w)+(\mathcal{A} \varphi) w
$$

Now we define the energy function for the system (5.1) as

$$
E(t) \equiv E(w, t)=\frac{1}{2} \int_{\Omega}\left(|\dot{w}|^{2}+a(x)|\mathcal{A} w|^{2}\right) d x .
$$


Then $E(t) \equiv E(0)$ for all $t>0$. Let

$$
L(t)=\int_{\Omega}\left(w^{2}+|D w|_{g}^{2}\right) d x
$$

be the lower-order term in terms of $E(t)$.

Lemma 5.5. Suppose that Assumption (H3) holds. Let $w$ solve the equation (5.1) with $\mathcal{A} w=0$ on $\Sigma_{0}$. Then $w \equiv 0$ in $Q$.

Proof. Set

$Y=\left\{w \in X \triangleq C\left([0, T] ; H_{0}^{2}(\Omega)\right) \cap C^{1}\left([0, T] ; L^{2}(\Omega)\right), w\right.$ solves $(2.1)$ with $\mathcal{A} w=0$ on $\left.\Sigma_{0}\right\}$.

We prove $Y=0$. Actually, by (5.3) and (5.40) that will be shown later, we have

$$
E(0) \leq C\left(\left\|\mathcal{A} w_{2}\right\|_{L^{2}\left(\Sigma_{0}\right)}^{2}+\|w\|_{L^{\infty}\left(0, T ; H_{0}^{1}(\Omega)\right)}^{2}\right), \forall w \in X \text { satisfying (5.1). }
$$

Now, we show that there exists a constant $C>0$ such that for any $w \in X$ satisfying $(5.1)$

$$
\|w\|_{L^{\infty}\left(0, T ; H_{0}^{1}(\Omega)\right)}^{2} \leq C\left(\left\|\mathcal{A} w_{2}\right\|_{L^{2}\left(\Sigma_{0}\right)}^{2}+\|w\|_{L^{\infty}\left(0, T ; L^{2}(\Omega)\right)}^{2}\right) .
$$

In fact, if (5.23) is not true, then there exists a solution sequence $\left\{w_{n}\right\} \in X$ to Equation (5.1) such that

$$
\left\|\mathcal{A} w_{n 2}\right\|_{L^{2}\left(\Sigma_{0}\right)}^{2}+\left\|w_{n}\right\|_{L^{\infty}\left(0, T ; L^{2}(\Omega)\right)}^{2} \rightarrow 0 \text { as } n \rightarrow \infty
$$

with

$$
\left\|w_{n}\right\|_{L^{\infty}\left(0, T ; H_{0}^{1}(\Omega)\right)}^{2}=1 .
$$

It then follows from (5.3) and (5.22) that $\left\{w_{n}\right\}$ is bounded in $X$ and hence is relatively compact in $L^{\infty}\left(0, T ; H_{0}^{1}(\Omega)\right)$. By extracting a subsequence if necessary, we may assume without loss of generality that $\left\{w_{n}\right\}$ converges strongly to $w \in L^{\infty}\left(0, T ; H_{0}^{1}(\Omega)\right)$. By $(5.25)$, it has

$$
\|w\|_{L^{\infty}\left(0, T ; H_{0}^{1}(\Omega)\right)}^{2}=1 .
$$

However, (5.24) implies $w=0$ in $Q$. This contradicts (5.26).

Next, from (5.22) and (5.23), we have

$$
E(w, 0) \leq C\left(\left\|\mathcal{A} w_{2}\right\|_{L^{2}\left(\Sigma_{0}\right)}^{2}+\|w\|_{L^{\infty}\left(0, T ; L^{2}(\Omega)\right)}^{2}\right), \forall w \in X \text { satisfying (5.1). }
$$

By the density argument, (5.27) still holds true for $w \in L^{\infty}\left(0, T ; L^{2}(\Omega)\right)$ satisfying (5.1). We thus have proved that $w \in Y$ implies that $v=\dot{w}$ solves (5.1) with $\left.\mathcal{A} v\right|_{\Sigma_{0}}=0$ and $v \in L^{\infty}\left(0, T ; L^{2}(\Omega)\right)$. This together with (5.27) gives

$$
(v(0), \dot{v}(0)) \in H_{0}^{2}(\Omega) \times L^{2}(\Omega) .
$$

Finally, since by Proposition 5.1, $v \in X$, it follows from (5.27) that the map $\partial_{t}: w \rightarrow \dot{w}$ is continuous from $Y$ to $Y$ and the injection of $\{w \in Y ; \dot{w} \in Y\}$ is compact. Therefore, $Y$ is a finite dimensional space. There must exist an $\eta \in \mathbb{C}$ and $w \in Y \backslash\{0\}$ such that $\dot{w}=\eta w$, which implies

$$
w(x, t)=e^{\eta t} w(x, 0)
$$

Substitute (5.29) into (5.1) to obtain (1.11) with $u(x)=w(x, 0)$ and $\zeta=-\eta^{2}$. By Assumption (H3) we have $w(x, t) \equiv 0$ and hence $Y=\{0\}$. 
With these preparations, we can now show Theorem 1.3.

Proof. By the duality principle, the proof is accomplished if we can show that there is a constant $C_{T}>0$ independent of the solution $w$ of Equation (5.1) such that

$$
E(0) \leq C_{T} \int_{\Sigma_{0}}\left(\mathcal{A} w_{2}\right)^{2} d \Sigma
$$

for $\Sigma_{0}=\Gamma_{0} \times(0, T)$, where $\Gamma_{0}$ given by (1.12) is relatively open in $\Gamma$.

To this end, for any $x \in \bar{\Omega}$ we define $T \in T^{2}\left(\mathbb{R}_{x}^{n}\right)$ by

$$
T(X, Y)=D N(X, Y)+D N(Y, X), \forall X, Y \in \mathbb{R}_{x}^{n}
$$

It is clear that $T(\cdot, \cdot)$ is symmetric, and from $(1.8)$, we have

$$
D N(X, Y)+D N(Y, X)=2 b(x)\langle X, Y\rangle_{g} \forall X, Y \in \mathbb{R}_{x}^{n}, x \in \bar{\Omega} .
$$

Given $x \in \bar{\Omega}$, let $\left\{e_{i}\right\}_{i=1}^{n}$ be an orthonormal basis of $\left(\mathbb{R}_{x}^{n}, g\right)$. By (5.32), we have

$$
\left\langle D N, D^{2} w\right\rangle_{T^{2}\left(\mathbb{R}_{x}^{n}\right)}=D N\left(e_{i}, e_{j}\right) D^{2} w\left(e_{i}, e_{j}\right)=b(x) \Delta_{g} w=b(x) \mathcal{A} w+D f(w) .
$$

By Lemma 5.1, there exist constants $C>0, \varepsilon>0$ such that

$$
\begin{gathered}
\left|\int_{\Omega} \dot{w} N(w) d x\right| \leq \varepsilon \int_{\Omega}|\dot{w}|^{2} d x+\frac{1}{4 \varepsilon} \int_{\Omega}|N(w)|^{2} d x \leq \varepsilon E(0)+\frac{C}{4 \varepsilon} L(t), \\
\left|\int_{\Omega} \dot{w} w \varphi d x\right| \leq \varepsilon E(0)+\frac{C}{4 \varepsilon} L(t) \\
\left|\int_{Q} a(x) \mathcal{A} w\left[\mathcal{F} N(w)+\operatorname{Ric}(N, D w)-D^{2} f(N, D w)\right] d Q\right| \\
\leq \varepsilon T E(0)+\frac{C}{4 \varepsilon} \int_{0}^{T} L(t) d t \\
\left|\int_{Q} a(x) b(x) \mathcal{A} w D f(w) d Q\right| \leq \varepsilon T E(0)+\frac{C}{4 \varepsilon} \int_{0}^{T} L(t) d t \\
\left|\int_{Q} a(x) \mathcal{A} w[w \mathcal{A} \varphi+2 D \varphi(w)] d Q\right| \leq \varepsilon T E(0)+\frac{C}{4 \varepsilon} \int_{0}^{T} L(t) d t .
\end{gathered}
$$


Keeping Assumptions (H1)-(H3) and $a_{2} \leq a_{1}$ in mind, by (5.8), (5.18) and (5.33)(5.38), we have

$$
\begin{aligned}
& \frac{1}{2} \int_{\Sigma_{0}} a_{2}\left(\mathcal{A} w_{2}\right)^{2} N \cdot \nu d \Sigma \\
\geq & \frac{1}{2} \int_{\Sigma} a_{2}\left(\mathcal{A} w_{2}\right)^{2} N \cdot \nu d \Sigma-\frac{1}{2} \int_{\Sigma_{1}}\left[a_{1}\left(\mathcal{A} w_{1}\right)^{2}-a_{2}\left(\mathcal{A} w_{2}\right)^{2}\right] N \cdot \nu d \Sigma \\
= & \left.\int_{\Omega} \dot{w} N(w) d x\right|_{0} ^{T}+\frac{1}{2} \int_{Q}\left[|\dot{w}|^{2}-a(x)(\mathcal{A} w)^{2}\right] \operatorname{div}_{0} N d Q \\
& +\int_{Q} a(x) \mathcal{A} w\left[2\left\langle D N, D^{2} w\right\rangle_{T^{2}\left(\mathbb{R}_{x}^{n}\right)}+\mathcal{F} N(w)+\operatorname{Ric}(N, D w)-D^{2} f(N, D w)\right] d Q \\
\geq & 2 b_{0} \int_{Q} a(x)(\mathcal{A} w)^{2} d Q+\frac{1}{2} \int_{Q}\left[|\dot{w}|^{2}-a(x)(\mathcal{A} w)^{2}\right] \operatorname{div}_{0} N d Q \\
& \quad-\left(2 \varepsilon E(0)+\frac{C}{4 \varepsilon}(L(T)+L(0))\right)-2\left(\varepsilon T E(0)+\frac{C}{4 \varepsilon} \int_{0}^{T} L(t) d t\right) \\
= & b_{0}\left(|\dot{w}|^{2}+a(x)(\mathcal{A} w)^{2}\right) d Q+\int_{Q}\left[|\dot{w}|^{2}-a(x)(\mathcal{A} w)^{2}\right]\left(\frac{1}{2} \operatorname{div} v_{0} N-b_{0}\right) d Q \\
& \quad-\left(2 \varepsilon E(0)+\frac{C}{4 \varepsilon}(L(T)+L(0))\right)-2\left(\varepsilon T E(0)+\frac{C}{4 \varepsilon} \int_{0}^{T} L(t) d t\right) \\
\geq & \left(b_{0}-4 \varepsilon-3 T \varepsilon\right) E(0)-\left(\frac{3 C}{4 \varepsilon} \int_{0}^{T} L(t) d t+\frac{C}{2 \varepsilon}(L(T)+L(0))\right) .
\end{aligned}
$$

Setting $\varepsilon>0$ small enough, we obtain

$$
E(0) \leq C_{T} \int_{\Sigma_{0}}(\mathcal{A} w)^{2} d \Sigma+C\left(\int_{0}^{T} L(t) d t+L(T)+L(0)\right) .
$$

Now we apply the standard compact uniqueness argument to absorb the lower-order terms in (5.40). In other words, we want to show that there exists a constant $C>0$ such that

$$
\|w\|_{L^{\infty}\left(0, T ; H_{0}^{1}(\Omega)\right)}^{2} \leq C \int_{\Sigma_{0}}(\mathcal{A} w)^{2} d \Sigma
$$

for solution $w$ of (5.1). In fact, if (5.41) is not true, then there exists a solution sequence $\left\{w_{n}\right\}$ of Equation (5.1) such that

$$
\int_{\Sigma_{0}}\left(\mathcal{A} w_{n}\right)^{2} d \Sigma \rightarrow 0, \quad n \rightarrow \infty
$$

with

$$
\left\|w_{n}\right\|_{L^{\infty}\left(0, T ; H_{0}^{1}(\Omega)\right)}^{2} \equiv 1 .
$$

It then follows from (5.40) and (5.3) that $\left\{w_{n}\right\}$ is a bounded sequence in $C\left([0, T] ; H_{0}^{2}(\Omega)\right) \cap$ $C^{1}\left([0, T] ; L^{2}(\Omega)\right)$ and hence relatively compact in $L^{\infty}\left(0, T ; H_{0}^{1}(\Omega)\right)$ because the injection

$$
C\left([0, T] ; H_{0}^{2}(\Omega)\right) \cap C^{1}\left([0, T] ; L^{2}(\Omega)\right) \rightarrow L^{\infty}\left(0, T ; H_{0}^{1}(\Omega)\right)
$$


is compact due to Simon's result [26]. By extracting a subsequence if necessary, we may assume without loss of generality that $\left\{w_{n}\right\}$ converges strongly to $w \in L^{\infty}\left(0, T ; H_{0}^{1}(\Omega)\right)$. By (5.43),

$$
\|w\|_{L^{\infty}\left(0, T ; H_{0}^{1}(\Omega)\right)}^{2}=1 .
$$

Furthermore, $\left\{w_{n}\right\}$ and $\left\{\dot{w}_{n}\right\}$ converge to $w$ and $\dot{w}$ in $L^{\infty}\left(0, T ; H_{0}^{2}(\Omega)\right)$ and $L^{\infty}\left(0, T ; L^{2}(\Omega)\right)$, respectively, in weak star topology. Therefore, $w$ is a solution to $(5.1)$ with

$$
w \in C\left([0, T] ; H_{0}^{2}(\Omega)\right) \cap C^{1}\left([0, T] ; L^{2}(\Omega)\right) .
$$

By (5.40) and (5.17), we have

$$
\mathcal{A} w=0 \text { on } \Sigma_{0}
$$

Finally, by virtue of Lemma 5.5, we have

$$
w \equiv 0 \text { in } Q
$$

which contradicts (5.44). The proof is complete.

Appendix. Since there is no explanation for the identity (2.8) in [34], here we give a brief proof.

Proof. Einstein summation is used throughout the proof . Let $x \in \mathbb{R}^{n}$ be fixed. Let $\left\{e_{i}\right\}_{i=1}^{n}$ be a frame field normal at the point $x$ on Riemannian manifold $\left(\mathbb{R}^{n}, g\right)$. Then at $x$, it has

$$
\begin{aligned}
\left\langle D N, D^{2} \varphi\right\rangle_{T^{2}\left(\mathbb{R}_{x}^{n}\right)} & =D N\left(e_{i}, e_{j}\right) D^{2} \varphi\left(e_{i}, e_{j}\right) \\
& =\left\langle D_{e_{i}} N, e_{j}\right\rangle_{g}\left[e_{i} e_{j}(\varphi)-D_{e_{i}} e_{j}(\varphi)\right] \\
& =\left\langle D_{e_{i}} N, e_{j}\right\rangle_{g} e_{i}\left\langle\nabla_{g} \varphi, e_{j}\right\rangle_{g} \\
& =\left\langle D_{e_{i}} N, e_{j}\right\rangle_{g}\left(\left\langle D_{e_{i}} \nabla_{g} \varphi, e_{j}\right\rangle_{g}+\left\langle\nabla_{g} \varphi, D_{e_{i}} e_{j}\right\rangle_{g}\right) \\
& =\left\langle D_{e_{i}} N, e_{j}\right\rangle_{g}\left\langle D_{e_{i}} \nabla_{g} \varphi, e_{j}\right\rangle_{g} \\
& =\left\langle D_{e_{i}} N, D_{e_{i}} \nabla_{g} \varphi\right\rangle_{g}
\end{aligned}
$$

By the formulae $\operatorname{div}_{g} X=\left\langle D_{e_{i}} X, e_{i}\right\rangle_{g}$ for any $X \in \mathfrak{X}\left(\mathbb{R}^{n}\right), \nabla_{g} \varphi=e_{i}(\varphi) e_{i}$ and $\left|\nabla_{g} \varphi\right|_{g}^{2}=\sum_{i=1}^{n}\left(e_{i}(\varphi)\right)^{2}$, we have, at $x$

$$
\begin{aligned}
N\left(\Delta_{g} \varphi\right) & =N\left(\operatorname{div}_{g}\left(\nabla_{g} \varphi\right)\right)=N\left\langle D_{e_{i}} \nabla_{g} \varphi, e_{i}\right\rangle_{g} \\
& =\left\langle\nabla_{g}\left\langle D_{e_{i}} \nabla_{g} \varphi, e_{i}\right\rangle_{g}, N\right\rangle_{g} \\
& =\left\langle\left(e_{j}\left\langle D_{e_{i}} \nabla_{g} \varphi, e_{i}\right\rangle_{g}\right) e_{j}, N\right\rangle_{g} \\
& =\left\langle\left\langle D_{e_{j}} D_{e_{i}}\left(\nabla_{g} \varphi\right), e_{i}\right\rangle_{g} e_{j}, N\right\rangle_{g} \\
& =\left\langle D_{e_{i}} D_{e_{i}}\left(\nabla_{g} \varphi\right), N\right\rangle_{g}
\end{aligned}
$$


Computing the first term on the right hand side of (2.8) we obtain, at $x$,

$$
\begin{aligned}
(-\triangle N)(\varphi) & =-\left(-D_{e_{k} e_{k}}^{2} N+e^{k} \wedge i\left(w^{j}\right) R\left(e_{k}, e_{j}\right) N\right)(\varphi)(\text { by the formula }(2.7)) \\
& =D_{e_{k}} D_{e_{k}} N(\varphi)-e^{k} \wedge i\left(w^{j}\right)\left\{\left\langle R\left(e_{k}, e_{j}\right) N, e_{l}\right\rangle_{g} e_{l}\right\}(\varphi) \\
& =\left\langle\nabla_{g} \varphi, D_{e_{k}} D_{e_{k}} N\right\rangle_{g}-\left\langle R\left(e_{k}, e_{j}\right) N, e_{j}\right\rangle_{g} e_{k}(\varphi) \\
& =\left\langle\nabla_{g} \varphi, D_{e_{k}} D_{e_{k}} N\right\rangle_{g}-\left\langle R\left(\nabla_{g} \varphi, e_{j}\right) N, e_{j}\right\rangle_{g} \\
& =\left\langle\nabla_{g} \varphi, D_{e_{k}} D_{e_{k}} N\right\rangle_{g}-\operatorname{Ric}\left(\nabla_{g} \varphi, N\right) .
\end{aligned}
$$

The left hand side of (2.8) at $x$ can be expressed as

$$
\begin{aligned}
\Delta_{g}(N(\varphi)) & =\operatorname{div}_{g}\left(\nabla_{g}(N(\varphi))\right)=\operatorname{div}_{g}\left(e_{i}(N(\varphi)) e_{i}\right) \\
& =\left\langle D_{e_{j}} e_{i}(N(\varphi)) e_{i}, e_{j}\right\rangle_{g} \\
& =e_{i} e_{i}\left\langle\nabla_{g} \varphi, N\right\rangle_{g} \\
& =e_{i}\left\{\left\langle D_{e_{i}} \nabla_{g} \varphi, N\right\rangle_{g}+\left\langle\nabla_{g} \varphi, D_{e_{i}} N\right\rangle_{g}\right\} \\
& =\left\langle D_{e_{i}} D_{e_{i}}\left(\nabla_{g} \varphi\right), N\right\rangle_{g}+2\left\langle D_{e_{i}} \nabla_{g} \varphi, D_{e_{i}} N\right\rangle_{g}+\left\langle\nabla_{g} \varphi, D_{e_{i}} D_{e_{i}} N\right\rangle_{g}
\end{aligned}
$$

Combining identities (A.1)-(A.4), noting the arbitrariness of $x$ and the fact that the Ricci curvature tensor $\operatorname{Ric}(\cdot, \cdot)$ is a symmetric second-order covariant tensor field, we finally obtain (2.8).

\section{REFERENCES}

[1] K. Ammari, Dirichlet boundary stabilization of the wave equation, Asymptotic Analysis, 30 (2002), 117-130. MR1919338 (2003f:93072)

[2] K. Ammari and M. Tucsnak, Stabilization of second order evolution equations by a class of unbounded feedbacks, ESAIM Control Optim. Calc. Var., 6 (2001), 361-386. MR1836048 (2002f:93104)

[3] M. Aassila, Exact boundary controllability of the plate equation, Differential Integral Equations, 13 (2000), 1413-1428. MR1787074 (2002e:93012)

[4] C. I. Byrnes, D. S. Gilliam, V. I. Shubov and G. Weiss, Regular linear systems governed by a boundary controlled heat equation, Journal of Dynamical and Control Systems, 8 (2002), 341-370. MR1914447 (2003d:93045)

[5] S. G. Chai and K. Liu, Boundary stabilization of the transmission of wave equations with variable coefficients, Chinese Ann. Math. Ser. A, 26(5) (2005), 605-612 (in Chinese). Translation in Chinese J. Contemp. Math., 26 (2005), no. 4, 337-346. MR2186628 (2006f:93089)

[6] S. G. Chai, Stabilization of thermoelastic plates with variable coefficients and dynamical boundary control, Indian J. Pure Appl. Math., 36 (2005), 227-249. MR2179402

[7] S. G. Chai, Boundary feedback stabilization of Naghdi's model, Acta Math. Sin. (Engl. Ser.), 21(1) (2005), 169-184. MR2128833 (2005k:93171)

[8] R. F. Curtain, The Salamon-Weiss class of well-posed infinite dimensional linear systems: A survey, IMA J. of Math. Control and Inform., 14 (1997), 207-223. MR1470034 (99a:93054)

[9] R. F. Curtain, Linear operator inequalities for strongly stable weakly regular linear systems, Math. Control Signals Systems, 14 (2001), 299-337. MR1868533 (2002k:93022)

[10] R. F. Curtain and G. Weiss, Well-posedness of triples of operators (in the sense of linear systems theory), in Control and Estimation of Distributed Parameter Systems (F. Kappel, K. Kunisch and W. Schappacher, Eds.), Vol. 91, Birkhäuser, Basel, 1989, 41-59. MR1033051 (91d:93027)

[11] B. Z. Guo and Y. H. Luo, Controllability and stability of a second order hyperbolic system with colocated sensor/actuator, Systems and Control Letters, 46 (2002), 45-65. MR2011071 (2004i:93015)

[12] B. Z. Guo and X. Zhang, The regularity of the wave equation with partial Dirichlet control and colocated observation, SIAM J. Control Optim., 44 (2005), 1598-1613. MR2193497 (2006j:93058) 
[13] B. Z. Guo and Z. C. Shao, Regularity of a Schrödinger equation with Dirichlet control and colocated observation, Systems and Control Letters, 54 (2005), 1135-1142. MR2170295 (2006d:35208)

[14] B. Z. Guo and Z. C. Shao, Regularity of an Euler-Bernoulli plate equation with Neumann control and colocated observation, J. Dyn. Control Syst., 12 (2006), no. 3, 405-418. MR2233027 (2007b:93076)

[15] B. Z. Guo and Z. X. Zhang, On the well-posedness and regularity of wave equations with variable coefficients and partial boundary Dirichlet control and colocated observation, ESAIM Control Optim. Calc. Var., to appear.

[16] B. Z. Guo and Z. X. Zhang, Well-posedness and regularity for an Euler-Bernoulli plate with variable coefficients and boundary control and observation, Mathematics of Control, Signals, and Systems, to appear.

[17] John E. Lagnese, Recent progress in exact boundary controllability and uniform stabilizability of thin beams and plates, in Distributed Parameter Control Systems, Lecture Notes in Pure and Appl. Math., 128, Dekker, New York, 1991, 61-111. MR1108855 (92f:93019)

[18] John E. Lagnese, Boundary controllability in problems of transmission for a class of second order hyperbolic systems, ESAIM Control Optim. Calc. Var., 2 (1991), 343-357. MR1487483 (98k:35114)

[19] I. Lasiecka and R. Triggiani, $L^{2}(\Sigma)$-regularity of the boundary to boundary operator $B^{*} L$ for hyperbolic and Petrowski PDEs, Abstr. Appl. Anal., No. 19, 2003, 1061-1139. MR2041290 (2005i:35164)

[20] J. L. Lions and E. Magenes, Non-Homogeneous Boundary Value Problems and Applications, Vol. I, Springer-Verlag, Berlin, 1972. MR0350177 (50:2670)

[21] W. Liu and G. H. Williams, Exact controllability for problems of transmission of the plate equation with lower order terms, Quart. Appl. Math., 58 (2000), 37-68. MR1738557 (2001d:93015)

[22] W. Liu and G. H. Williams, The exponential stability of the problem of transmission of the wave equation, Bull. Austra. Math. Soc., 57 (1998), 305-327. MR1617324 (99b:35112)

[23] H. P. Oquendo, Nonlinear boundary stabilization for a transmission problem in elasticity, Nonlinear Analysis, 52 (2003), 1331-1345. MR1941260 (2003i:93069)

[24] D. L. Russell, Exact boundary value controllability theorems for wave and heat processes in starcomplemented regions, in Differential Games and Control Theory (Roxin, Liu and Sternberg, Eds.), Lecture Notes in Pure Appl. Math., 10, Marcel Dekker, New York, 1974, 291-319. MR0467472 (57:7329)

[25] D. L. Russell, Controllability and stabilizability theory for linear partial differential equations: Recent progress and open questions, SIAM Review, 20 (1978), no. 4, 639-739. MR508380 (80c:93032)

[26] J. Simon, Compact sets in the space $L^{p}(0, T ; B)$, Ann. Mat. Pura. Appl., 146(4) (1987), 65-96. MR916688 (89c:46055)

[27] O. J. Staffans, Passive and conservative continuous-time impedance and scattering systems. Part I: well-posed systems, Math. Control, Signals, and Systems, 15 (2002), 291-315. MR1942089 (2003i:93024)

[28] M. E. Taylor, Partial Differential Equations I: Basic Theory, Springer-Verlag, New York, 1996. MR1395148 (98b:35002b)

[29] G. Weiss, Transfer functions of regular linear systems I: characterizations of regularity, Trans. Amer. Math. Soc., 342 (1994), 827-854. MR1179402 (94f:93074)

[30] G. Weiss and R. Rebarber, Optimizability and estimatability for infinite-dimensional linear systems, SIAM J. Control Optim., 39 (2000), 1204-1232. MR1814273 (2001m:93021)

[31] G. Weiss, O. J. Staffans and M. Tucsnak, Well-posed linear systems-a survey with emphasis on conservative systems, Int. J. Appl. Math. Comput. Sci., 11 (2001), 7-33. MR1835146 (2002f:93068)

[32] H. Wu, Bochner's skills in differential geometry (Part I), Advances in Mathematics, Vol. 10, No. 1 (1981), 57-76 (Chinese). MR691910 (84m:53054)

[33] H. Wu, C. L. Shen and Y. L. Yu, An Introduction to Riemannian Geometry, Beijing University Press, Beijing, 1989 (in Chinese).

[34] P. F. Yao, Observability inequalities for the Euler-Bernoulli plate with variable coefficients, Contemporary Mathematics, Vol. 268, Amer. Math. Soc., Providence, RI, 2000, 383-406. MR1804802 (2001m:93025)

[35] P. F. Yao, On the observability inequality for exact controllability of wave equations with variable coefficients, SIAM J. Contr. and Optim., 37 (1999) 1568-1599. MR1710233 (2000m:93027) 